This item was submitted to Loughborough's Research Repository by the author.

Items in Figshare are protected by copyright, with all rights reserved, unless otherwise indicated.

\title{
Model colloidal fluid with competing interactions: bulk and interfacial properties
}

PLEASE CITE THE PUBLISHED VERSION

http://dx.doi.org/10.1063/1.2405355

PUBLISHER

(c) American Institute of Physics

VERSION

VoR (Version of Record)

LICENCE

CC BY-NC-ND 4.0

REPOSITORY RECORD

Archer, Andrew J., D. Pini, Robert Evans, and L. Reatto. 2019. "Model Colloidal Fluid with Competing Interactions: Bulk and Interfacial Properties". figshare. https://hdl.handle.net/2134/16022. 


\section{AIP | The Journal of Chemical Physics}

Model colloidal fluid with competing interactions: Bulk and interfacial properties

A. J. Archer, D. Pini, R. Evans, and L. Reatto

Citation: The Journal of Chemical Physics 126, 014104 (2007); doi: 10.1063/1.2405355

View online: http://dx.doi.org/10.1063/1.2405355

View Table of Contents: http://scitation.aip.org/content/aip/journal/jcp/126/1 ?ver=pdfcov

Published by the AIP Publishing

\section{Articles you may be interested in}

Fluid phase behavior of a model colloid-polymer mixture: Influence of polymer size and interaction strength J. Chem. Phys. 129, 164907 (2008); 10.1063/1.3000011

Microstructure and depletion forces in polymer-colloid mixtures from an interfacial statistical associating fluid theory

J. Chem. Phys. 128, 164910 (2008); 10.1063/1.2909975

Surface properties of fluids of charged platelike colloids

J. Chem. Phys. 125, 184704 (2006); 10.1063/1.2360939

Interfacial statistical geometry: Fluids adsorbed in wedges and at edges

J. Chem. Phys. 120, 1535 (2004); 10.1063/1.1634253

Bulk and interfacial properties of quadrupolar fluids

J. Chem. Phys. 117, 3982 (2002); 10.1063/1.1495841

\section{AIP $\mid$ chaos CALL FOR APPLICANTS Seeking new Editor-in-Chief}




\title{
Model colloidal fluid with competing interactions: Bulk and interfacial properties
}

\author{
A. J. Archer ${ }^{a)}$ \\ H. H. Wills Physics Laboratory, University of Bristol, Bristol BS8 1TL, United Kingdom \\ D. Pini \\ Dipartimento di Fisica, Università degli Studi di Milano, Via Celoria 16, 20133 Milano, Italy \\ R. Evans \\ H. H. Wills Physics Laboratory, University of Bristol, Bristol BS8 1TL, United Kingdom \\ L. Reatto \\ Dipartimento di Fisica, Università degli Studi di Milano, Via Celoria 16, 20133 Milano, Italy
}

(Received 10 October 2006; accepted 14 November 2006; published online 4 January 2007)

\begin{abstract}
Using a simple mean field density functional theory (DFT), the authors investigate the structure and phase behavior of a model colloidal fluid composed of particles interacting via a pair potential which has a hard core of diameter $\sigma$, is attractive Yukawa at intermediate separations, and is repulsive Yukawa at large separations. The authors analyze the form of the asymptotic decay of the bulk fluid correlation functions, comparing results from DFT with those from the self-consistent Ornstein-Zernike approximation (SCOZA). In both theories the authors find rich crossover behavior, whereby the ultimate decay of correlation functions changes from monotonic to long wavelength damped oscillatory decay on crossing certain lines in the phase diagram or sometimes from oscillatory to oscillatory with a longer wavelength. For some choices of potential parameters the authors find, within the DFT, a $\lambda$ line at which the fluid becomes unstable with respect to periodic density fluctuations. SCOZA fails to yield solutions for state points near such a $\lambda$ line. The propensity towards clustering of particles, which is reflected by the presence of a long wavelength $(\gg \sigma)$ slowly decaying oscillatory pair correlation function, and a structure factor that exhibits a very sharp maximum at small but nonzero wave numbers, is enhanced in states near the $\lambda$ line. The authors present density profiles for the planar liquid-gas interface and for fluids adsorbed at a planar hard wall. The presence of a nearby $\lambda$ transition gives rise to pronounced long wavelength oscillations in the one-body density profiles at both types of interface. (C) 2007 American Institute of Physics. [DOI: 10.1063/1.2405355]
\end{abstract}

\section{INTRODUCTION}

Recently, colloidal systems have been synthesized in which the effective pair potential between the colloidal particles is attractive just outside the core separation distance, but is repulsive for larger particle separations. ${ }^{1-6}$ The longrange repulsion stems from the colloids being charged, and the short range attraction is generated by the depletion mechanism, ${ }^{7}$ arising from the addition of nonadsorbing polymers to the solution. ${ }^{8}$ Such competing interactions can give rise to phase behavior that can be very different from that found in "simple" liquids. Theory and simulation in both two and three dimensions for model systems with competing interactions predict that such interactions can give rise to a state with undamped periodic density fluctuations, which indicates a transition to cluster or striped phases (microphase separation). ${ }^{9-18}$ In the cluster phase the colloids are ordered in such a way that there are assemblies containing tens or hundreds of particles and large voids between the clusters

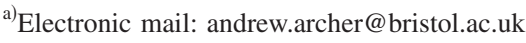

containing hardly any particles. Similarly, in the striped phase in two dimensions the particles are arranged in parallel stripes with low-density regions in between the stripes. $2,14,18$ In three dimensions the stripes form a gel-like network of elongated clusters. ${ }^{5,15,17}$ This behavior is most striking given that the pair interactions between the particles are spherically symmetrical and suggests that such systems could be important candidates for developing self-assembling pattern forming materials. The liquid-vapor phase transition may be preempted by a transition to a cluster or stripe phase, but if the long-range repulsion is not sufficiently strong, no permanent clusters or stripes are present, and a liquid-vapor phase transition is found. However, the liquid-gas coexistence curve is unusually flat in the critical region. ${ }^{19}$

While the bulk phase behavior has begun to be understood, there are no detailed studies of how the long-range decay of the pair correlation functions is affected by the competition between attraction and repulsion and very little is known about the properties of such fluids in inhomogeneous situations where the average fluid density is nonuniform. In the present paper we present a mean field density 
functional theory (DFT) for a simple model of such systems which provides a means of analyzing correlation functions and a first step in elucidating the properties of inhomogeneous fluids composed of particles with competing interactions.

The particular model fluid we consider in this paper is that described in Refs. 19-23, in which particles interact via a double-Yukawa pair potential of the following form:

$$
\beta v(r)= \begin{cases}\infty, & r \leqslant \sigma \\ -\epsilon \sigma \exp \left(-Z_{1}(r / \sigma-1)\right) / r+A \sigma \exp \left(-Z_{2}(r / \sigma-1)\right) / r, & r>\sigma,\end{cases}
$$

where $\beta=1 / k_{B} T$ is the inverse temperature (we shall set $\beta$ $=1), \sigma$ is the diameter of the particles, and the amplitudes $\epsilon>0$ and $A>0$. For a fixed value of $A$, the parameter $\epsilon$ plays a role somewhat akin to an inverse temperature. In the context of colloid-polymer mixtures one can envisage an athermal system at a fixed temperature and varying the chemical potential of a polymer reservoir, thereby changing the strength of the depletion attraction. Indeed, in the simple Asakura-Oosawa-Vrij model, the depletion attraction between colloidal particles arising from the exclusion of ideal polymers, yields a well depth proportional to $z_{p}$, the fugacity of the ideal polymers. ${ }^{24-26}$ Following the spirit of this approach, we can view our parameter $\epsilon$ as a measure of the polymer fugacity. In much of the work described here we follow Pini et al. ${ }^{19,20}$ by setting the two (dimensionless) decay lengths $Z_{1}=1$ and $Z_{2}=0.5$. However, we also present some results for other choices of $Z_{1}$ and $Z_{2}$.

A number of phase diagrams for this model system are displayed in Refs. 19 and 20, for cases when $A$ is not too large, i.e., cases when the repulsive contribution to the pair potential $v(r)$ is not sufficiently large that the liquid-gas phase separation is replaced by microphase separation. For these cases one finds that there can be a substantial supercritical region in the phase diagram where the compressibility is unusually large. ${ }^{19,20}$ In Refs. 19-23 a number of theories have been used to investigate the bulk fluid correlation functions [radial distribution function $g(r)$ and structure factor $S(k)$ ]. Here we make a systematic study of the asymptotic decay $r \rightarrow \infty$ of $g(r)$ in various regions of the phase diagram. The form of the decay of $g(r)$ is determined by the poles of $S(k)$ in the upper half of the complex $k$ plane. ${ }^{27}$ We use a simple DFT, equivalent to the random phase approximation (RPA) of the tail potential outside the hard core for the bulk fluid, to elucidate the pole structure for particles interacting with pair potentials given by Eq. (1). Due to the competing interactions, the fluid displays a propensity towards cluster formation which is manifested for some state points as a slowly decaying, long wavelength oscillatory decay of the radial distribution function $g(r)$ - the wavelength being related to the size of the clusters. Of course, at sufficiently low densities the decay of $g(r)$ is monotonic. We locate lines in the phase diagram at which the ultimate asymptotic decay of $g(r)$ crosses over from monotonic to oscillatory. In addition we find, for some values of the fluid pair potential parameters, that there can be a crossover in the decay of $g(r)$ from long wavelength oscillatory to oscillatory with wavelength $\sim \sigma$. We find that the pole structure displayed by our simple (RPA) DFT, and hence the crossover behavior, is mimicked closely by the more accurate self-consistent Ornstein-Zernike approximation (SCOZA) ${ }^{28,29}$ indicating that the simple DFT describes, at least qualitatively, the main features of the fluid structure.

For sufficiently large values of $A$ we find that on increasing $\epsilon$, the peak in the structure factor calculated from the DFT can diverge at $k=k_{c} \neq 0$. We denote the line in the phase diagram at which this divergence occurs as the " $\lambda$ line." $" 30-32$ On the $\lambda$ line the fluid becomes unstable with respect to periodic density fluctuations with wave number $k_{c}$, indicating that there is a phase transition to a modulated phase (either a crystalline or striped phase), which may be preempted by a transition to a glassy nonergodic state. ${ }^{15,21,33}$ In mean field treatments the $\lambda$ line encloses a region of the phase diagram where one would expect to find the liquid-gas critical point, and it intersects the binodal at densities on either side of the critical point. ${ }^{10}$ We find such behavior in the present DFT approach

This paper is laid out as follows: In Sec. II we introduce our simple DFT approach, and in Sec. III we describe briefly the implementation of the more accurate (bulk) SCOZA theory for determining correlation functions and phase behavior. In Sec. IV we present results for the bulk phase behavior for a range of pair potential parameters. Section V describes our approach for determining the ultimate asymptotic decay of the radial distribution functions and presents a number of representative results. In Sec. VI we apply our DFT to calculate inhomogeneous fluid profiles at a planar hard wall and at the liquid-gas planar free interface. We conclude in Sec. VII with a summary and discussion of our results.

\section{A MEAN FIELD DENSITY FUNCTIONAL THEORY}

The basis of DFT is that there exists a functional, $\Omega_{V}[\rho]$, of the fluid one-body density profile $\rho(\mathbf{r})$ such that the fluid equilibrium profile $\rho(\mathbf{r})$ minimizes this functional, 


$$
\left.\frac{\delta \Omega_{V}\left[\rho^{\prime}\right]}{\delta \rho^{\prime}(\mathbf{r})}\right|_{\rho^{\prime}(\mathbf{r})=\rho(\mathbf{r})}=0,
$$

and the minimal value of this functional is the grand potential $\Omega$ of the fluid. ${ }^{34}$ The grand potential functional $\Omega_{V}$ can be written as

$$
\Omega_{V}[\rho]=\mathcal{F}[\rho]-\int \mathrm{d} \mathbf{r} \rho(\mathbf{r})\left[\mu-V_{\mathrm{ext}}(\mathbf{r})\right],
$$

where $\mu$ is the chemical potential, $V_{\text {ext }}(\mathbf{r})$ is a one-body external potential, and $\mathcal{F}[\rho]$, the intrinsic Helmholtz free energy functional, is a unique functional of $\rho(\mathbf{r})$ for a given interaction potential. We can write $\mathcal{F}[\rho]=\mathcal{F}_{\text {id }}[\rho]+\mathcal{F}_{\text {ex }}[\rho]$, where

$$
\mathcal{F}_{\text {id }}[\rho]=k_{B} T \int \mathrm{d} \mathbf{r} \rho(\mathbf{r})\left[\ln \left(\rho(\mathbf{r}) \Lambda^{3}\right)-1\right]
$$

is the ideal-gas contribution, $\Lambda$ is the thermal de Broglie wavelength, and $\mathcal{F}_{\text {ex }}$ is the (unknown) excess contribution. Taking two functional derivatives of $\mathcal{F}_{\text {ex }}$, we obtain the (Ornstein-Zernike) pair direct correlation function for the inhomogeneous fluid, ${ }^{34}$

$$
c^{(2)}\left(\mathbf{r}, \mathbf{r}^{\prime}\right)=-\beta \frac{\delta^{2} \mathcal{F}_{\mathrm{ex}}[\rho]}{\delta \rho\left(\mathbf{r}^{\prime}\right) \delta \rho(\mathbf{r})} .
$$

In the present work we approximate the excess Helmholtz free energy functional by

$$
\mathcal{F}_{\text {ex }}[\rho]=\mathcal{F}_{\text {ex }}^{\mathrm{hs}}[\rho]+\frac{1}{2} \int \mathrm{d} \mathbf{r} \int \mathrm{d} \mathbf{r}^{\prime} \rho(\mathbf{r}) \rho\left(\mathbf{r}^{\prime}\right) v_{p}\left(\left|\mathbf{r}-\mathbf{r}^{\prime}\right|\right),
$$

where $\mathcal{F}_{\text {ex }}^{\text {hs }}[\rho]$ is the reference hard sphere Helmholtz excess free energy functional and the contribution due to the remainder of the pair potential $>\sigma$ is treated in a mean field fashion. ${ }^{34}$ We define the "perturbation" potential as

$$
\beta v_{p}(r)= \begin{cases}-\epsilon+A, & r \leqslant \sigma \\ \beta v(r), & r>\sigma .\end{cases}
$$

Note that $-\epsilon+A$ is the value of $\beta v(r)$ at contact, i.e., at $r$ $=\sigma^{+}$. We employ the Rosenfeld fundamental measure theory ${ }^{35-37}$ for $F_{\mathrm{ex}}^{\mathrm{hs}}[\rho]$. This nonlocal functional, via Eq. (5), generates $c_{\mathrm{PY}}\left(r, \rho_{b}\right)$, the Percus-Yevick (PY) pair direct correlation function for a uniform hard sphere fluid. Thus, in bulk, Eqs. (5) and (6) together generate the following simple approximation (RPA) for the pair direct correlation function:

$$
c^{(2)}\left(r ; \rho_{b}\right)=c_{\mathrm{PY}}\left(r ; \rho_{b}\right)-\beta v_{p}(r),
$$

where $\rho_{b}$ is the bulk fluid density. As in other RPA treatments of models with hard cores, the perturbation potential is not defined uniquely within the hard core. We choose the form in Eq. (7) for simplicity and because the resulting phase diagrams are closer to those obtained from the SCOZA than those from other possible choices that we considered. With this choice the Fourier transform of $c(r) \equiv c^{(2)}\left(r ; \rho_{b}\right)$ can be carried out analytically and yields

$$
\hat{c}(k)=\hat{c}_{\mathrm{PY}}(k)-\beta \hat{v}(k),
$$

where $\hat{c}_{\mathrm{PY}}(k)$, the Fourier transform of $c_{\mathrm{PY}}\left(r ; \rho_{b}\right)$, is given by $^{38}$

$$
\begin{aligned}
\hat{c}_{\mathrm{PY}}(k)= & -4 \pi \sigma^{3}\left[\left(\frac{\alpha+2 \beta+4 \gamma}{q^{3}}-\frac{24 \gamma}{q^{5}}\right) \sin q\right. \\
& +\left(-\frac{\alpha+\beta+\gamma}{q^{2}}+\frac{2 \beta+12 \gamma}{q^{4}}-\frac{24 \gamma}{q^{6}}\right) \\
& \left.\times \cos q+\left(\frac{24 \gamma}{q^{6}}-\frac{2 \beta}{q^{4}}\right)\right],
\end{aligned}
$$

with $q=k \sigma$, and the coefficients

$$
\begin{aligned}
& \alpha=\frac{(1+2 \eta)^{2}}{(1-\eta)^{4}}, \\
& \beta=\frac{-6 \eta(1+\eta / 2)^{2}}{(1-\eta)^{4}}, \\
& \gamma=\frac{\eta(1+2 \eta)^{2}}{2(1-\eta)^{4}},
\end{aligned}
$$

depend on $\eta=\pi \rho_{b} \sigma^{3} / 6$, the packing fraction, and

$$
\begin{aligned}
\beta \hat{v}_{p}(k)= & -\frac{4 \pi \sigma^{3} \epsilon}{Z_{1}^{2}+q^{2}}\left(\cos q+\frac{Z_{1}}{q} \sin q\right) \\
& +\frac{4 \pi \sigma^{3} A}{Z_{2}^{2}+q^{2}}\left(\cos q+\frac{Z_{2}}{q} \sin q\right) \\
& -\frac{4 \pi \sigma^{3}(\epsilon-A)}{q^{3}}(\sin q-q \cos q) .
\end{aligned}
$$

Thus we have a relatively simple analytic expression for $\hat{c}(k)$ and therefore also for the static structure factor obtained from the Ornstein-Zernike relation, ${ }^{39}$

$$
S(k)=\frac{1}{1-\rho_{b} \hat{c}(k)} .
$$

In Fig. 1, we display $S(k)$ calculated at a bulk fluid density $\rho_{b} \sigma^{3}=0.2457$, the critical point density as obtained from DFT (see Sec. IV), for a number of different values of the parameter $\epsilon$, for the case when $A=0.082, Z_{1}=1$, and $Z_{2}$ $=0.5$. We see that as $\epsilon^{-1}$ is decreased, the structure factor develops a peak at a small, but nonzero, wave vector $k=k_{c}$ $\ll 2 \pi / \sigma$. This peak indicates the propensity towards clustering in the fluid. ${ }^{2,5,11,14,15,21-23}$ A similar RPA approximation was used in Ref. 14 to account for the structure factor of a two dimensional fluid of particles with competing interactions close to those of the present fluid. The propensity towards clustering in the fluid can also be seen in Fig. 2 where we plot the radial distribution function $g(r)$ for the same fluid at $\rho_{b} \sigma^{3}=0.2$ and several values of $\epsilon^{-1}$. We observe the development of long wavelength oscillations in $g(r)$ as $\epsilon^{-1}$ is decreased. These results are obtained from the inverse Fourier transform of $S(k)$. Note that within this route, the core condition that $g(r)=0$ for $r<\sigma$ is violated. This would not be the case if we were to use the test particle route to $g(r)$, i.e., if we treat one of the particles as a fixed external potential $\left[V_{\text {ext }}(\mathbf{r})=v(r)\right.$ in Eq. (3)], use the DFT to calculate the inhomogeneous fluid density profile, $\rho(r)$, around this fixed particle, and divide by the bulk density to obtain $g(r)=\rho(r) / \rho_{b}$. 


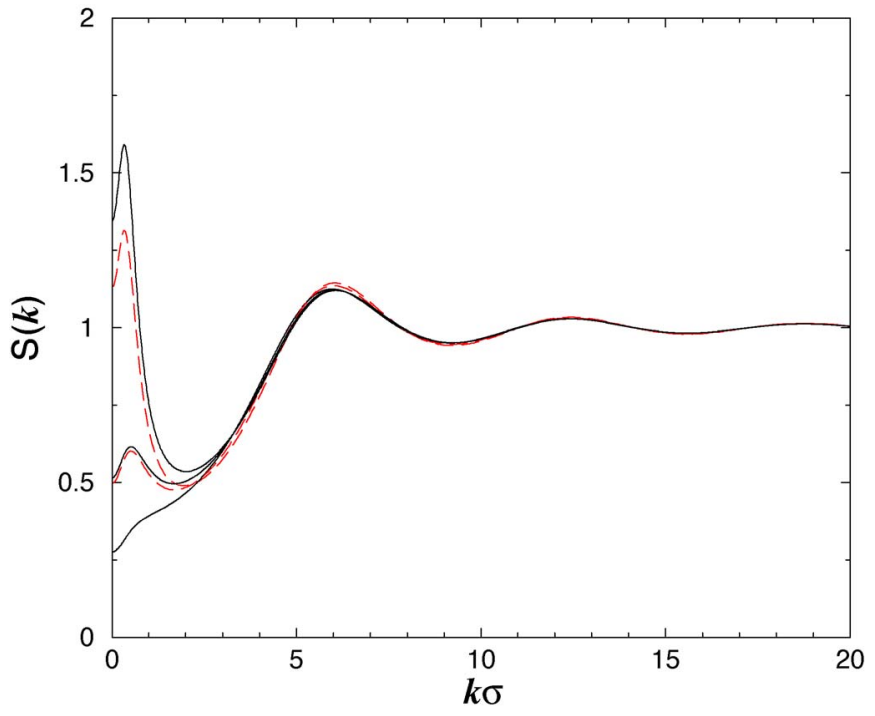

FIG. 1. Static structure factors $S(k)$ for a fluid with total density $\rho_{b} \sigma^{3}$ $=0.2457$, the critical point density determined from the DFT (RPA), and with the parameters $A=0.082, Z_{1}=1$, and $Z_{2}=0.5$, calculated for $\epsilon^{-1}=10,3$, and 2 (bottom to top). The solid lines are the results of the DFT (or RPA) [Eqs. (8)-(13)], and the dashed lines from SCOZA (there is no SCOZA result for $\epsilon^{-1}=10$ ). Note that as $\epsilon^{-1}$ is decreased, a peak develops and grows at a small wave vector $k=k_{c} \neq 0$, whereas the other peaks in $S(k)$, determined primarily by correlations in the reference hard sphere fluid, are almost unchanged.

For sufficiently large values of $A$ we find that on decreasing $\epsilon^{-1}$ at fixed $\rho_{b}$ the peak in the structure factor that occurs at small $k=k_{c} \neq 0$ can diverge. The line in the phase diagram at which this occurs is the $\lambda$ line..$^{30-32}$ This line is shown in the inset of Fig. 5 for the case where $A=0.082$. Inside the region bounded by the $\lambda$ line, the RPA result for $S(k)$ is unphysical, since this becomes negative in a certain interval of $k$, and goes from negative to positive divergent values upon crossing the boundaries of the interval.

\section{THE SCOZA FOR CORRELATION FUNCTIONS AND THERMODYNAMICS}

The SCOZA is designed to deal with two-body potentials which, like that of Eq. (1), consist of a singular hard sphere repulsive part, with diameter $\sigma$, and a longer-ranged tail. As is customary in integral equation theories, this approach is based on the Ornstein-Zernike (OZ) equation linking the radial distribution function $g(r)$ to the pair direct correlation function $c(r)$. A closed theory is obtained by supplementing the OZ equation with an approximate (closure) relation involving $g(r)$ and $c(r)$. In its simplest form, the SCOZA amounts to setting ${ }^{28}$

$$
\begin{cases}g(r)=0, & r \leqslant \sigma \\ c(r)=K\left(\rho_{b}, \beta\right) v(r), & r>\sigma .\end{cases}
$$

This closure differs from the RPA considered in Sec. II in two respects: first, the core condition on the radial distribution function is satisfied. Second, the amplitude $K$ of the direct correlation function outside the repulsive core has not been set to $K \equiv-\beta$. Rather, $K$ is regarded as an unknown state-dependent quantity, to be determined in such a way that consistency between the compressibility and the energy route

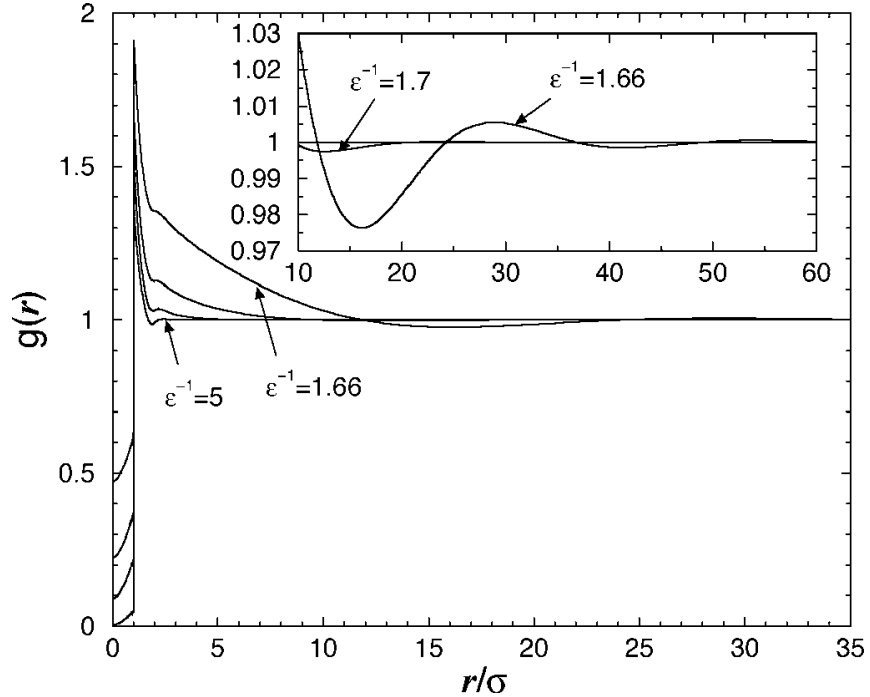

FIG. 2. Radial distribution function $g(r)$ for a fluid with total density $\rho_{b} \sigma^{3}=0.2$, with the parameters $A=0.082, Z_{1}=1$, and $Z_{2}=0.5$, calculated for $\epsilon^{-1}=5,2,1.7$, and 1.66. Note that as $\epsilon^{-1}$ is decreased, $g(r)$ becomes longer ranged; the decay is oscillatory, with a wavelength of about $25 \sigma$ for $\epsilon^{-1}$ $=1.66$ [a state close to the $\lambda$ line for this system (see Fig. 5); see the magnification in the inset]. Within the present DFT (RPA) treatment, based on Fourier transforming [Eq. (13)], the core condition $g(r)=0$ for $r<\sigma$ is violated (see text).

to thermodynamics is enforced. The previous applications of SCOZA, including the study of fluids with competing interactions, ${ }^{19,20}$ were aimed at thermal systems with a temperature-independent potential, such that the phase diagram can be plotted as a function of density and temperature. For thermal fluids, the consistency condition amounts to requiring that the reduced compressibility $\chi_{\text {red }}$ and the excess internal energy per unit volume $u$ satisfy the condition

$$
\frac{\partial}{\partial \beta}\left(\frac{1}{\chi_{\mathrm{red}}}\right)=\rho_{b} \frac{\partial^{2} u}{\partial \rho_{b}^{2}},
$$

where $\chi_{\text {red }}$ is obtained from the compressibility sum rule, i.e., $\chi_{\text {red }}=S(0)$, while $u$ is obtained from the energy equation $u=2 \pi \rho_{b}^{2} \int_{\sigma}^{\infty} \mathrm{d} r r^{2} v(r) g(r)$. If the closure [Eq. (14)] is implemented for the correlations, the consistency condition [Eq. (15)] yields a closed partial differential equation (PDE) for the function $K\left(\rho_{b}, \beta\right)$. It should be noted that in the present case $\beta v(r)$ is in effect independent of temperature [see Eq. (1)], and the phase diagrams will be plotted as a function of $\epsilon^{-1}$, the inverse strength of the attractive tail potential. For such an athermal system, Eq. (15) does not hold anymore, as a consequence of the trivial dependence on the temperature of the Helmholtz free energy, $F \sim k_{B} T$. However, for any given $\epsilon$, the value of $\beta F$ of the athermal system is the same as that given by a temperature-independent interaction $\widetilde{v}(r)$ $=w \beta v(r)$ at a temperature $k_{B} T=w$, where $\beta v(r)$ is given in Eq. (1) and $w$ is an arbitrary energy scale. For this system, Eq. (15) is valid. Therefore, the phase diagram and the correlations of the original potential [Eq. (1)] have been determined by integrating the SCOZA PDE for $\widetilde{v}(r)$ down to the reduced temperature $T^{*}=k_{B} T / w=1$ for each of the values of $\epsilon$ considered. Equivalently, one may reformulate Eq. (15) for the athermal system by replacing the inverse temperature $\beta$ 
with a coupling constant which varies from zero to unity, whose purpose is to switch on the tail interaction.

In the present case, implementing the SCOZA scheme is made simpler by taking advantage of the analytical results ${ }^{40}$ obtained for the mean spherical approximation (MSA) when the tail potential has a two-Yukawa form as in Eq. (1). These enable one to obtain $\chi_{\text {red }}$ as a function of $\rho_{b}$ and $u$. The function $\chi_{\text {red }}\left(\rho_{b}, u\right)$ can then be used in Eq. (15) by taking $u$ instead of $K$ as the unknown quantity. The algebraic manipulations have been described in detail in Ref. 41. The resulting PDE for $u$ supplemented with suitable initial and boundary conditions $^{29}$ is integrated numerically. Integration of $u$ with respect to $\beta$ then yields the Helmholtz free energy and hence all the other thermodynamic quantities.

The SCOZA, like the RPA, yields a spinodal curve, i.e., a locus in the temperature-density plane where the compressibility diverges. As soon as one enters the region bounded by the spinodal, $\chi_{\text {red }}$ is no longer positive, so that the theory ceases to be meaningful. Therefore, the region bounded by the spinodal must be excluded from the integration domain. If the temperature is below its critical value and one approaches the critical density $\rho_{c}$ either from $\rho=0$ or from the high-density boundary $\rho_{0}$, it is found that $\chi_{\text {red }}$ is no longer positive for $\rho=\rho_{1 s}$ or $\rho=\rho_{2 s}$, where $\rho_{1 s}$ and $\rho_{2 s}$ are temperature-dependent densities with $\rho_{1 s}<\rho_{c}<\rho_{2 s}$. The integration is then restricted to the intervals $\left(0, \rho_{1 s}-\Delta \rho\right)$ and $\left(\rho_{2 s}+\Delta \rho, \rho_{0}\right)$, where $\Delta \rho$ is the density spacing. Within the accuracy of the numerical discretization, $\rho_{1 s}$ and $\rho_{2 s}$ give the densities of the spinodal curve at the temperature considered. The liquid-gas coexistence curve is determined by equating the pressures and chemical potentials on the low- and highdensity branches of the subcritical isotherms.

\section{RESULTS FOR PHASE DIAGRAMS}

In this section we present results for the liquid-gas coexistence curve (binodal) and spinodal calculated from both theories. Within the present DFT it is straightforward to determine liquid-gas coexistence. By replacing $\rho(\mathbf{r})=\rho_{b}$ in the Helmholtz free energy functional [Eq. (6)], we obtain an expression for the bulk Helmholtz free energy per particle, $f$. The corresponding pressure can exhibit a van der Waals loop, and we calculate the coexisting gas and liquid densities in the standard way. We obtain the spinodal from the free energy as the locus of state points where $\partial^{2} f / \partial v^{2}=0$, where $v=1 / \rho_{b}$ is the specific volume, i.e., the locus where the isothermal compressibility diverges. This is identical to the locus of points where $S(k=0)$ diverges, where $S(k)$ is given by the Ornstein-Zernike relation [Eq. (13)] together with Eqs. (9)-(12). The self-consistency between the free energy calculated directly from the functional and from the compressibility route is one of the appealing features of the simple theory. In Figs. 3-6 we display the resulting DFT phase diagrams for $Z_{1}=1$ and $Z_{2}=0.5$ and a number of choices of the parameter $A$. In Figs. 4 and 5 we also display the corresponding SCOZA results for the spinodal and binodal. We find quite good agreement between the two theories although the

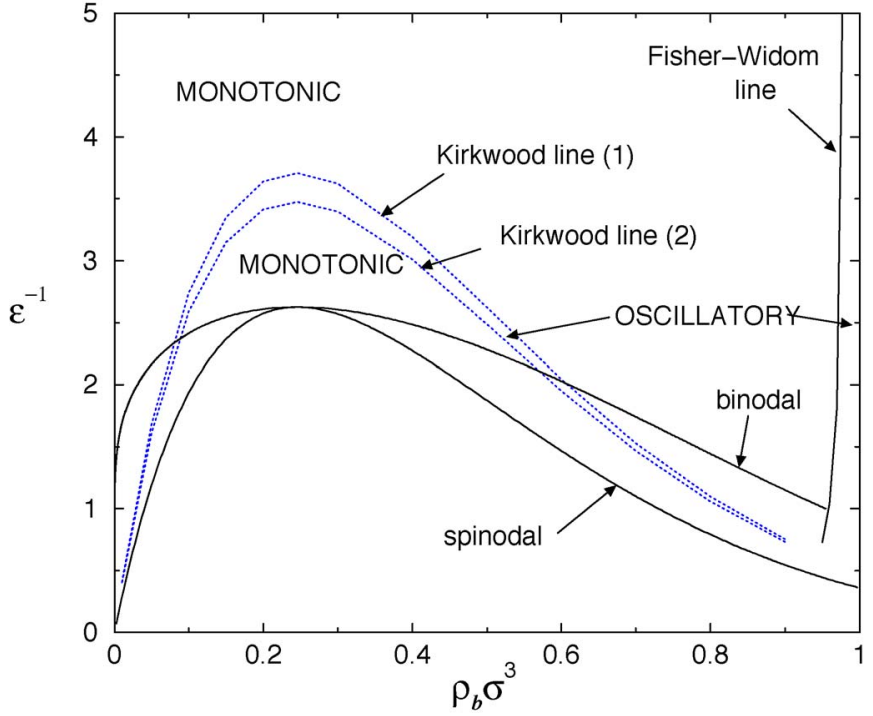

FIG. 3. Phase diagram for the case when $Z_{1}=1, Z_{2}=0.5$, and $A=0.0001$ obtained from the DFT (RPA). The solid lines denote the binodal and spinodal. The two dotted Kirkwood lines denote loci at which the asymptotic decay of $h(r)$ crosses over from monotonic to oscillatory. For the present choices of $Z_{1}$ and $Z_{2}$, the Fisher-Widom line is at densities $\rho_{b} \sigma^{3} \gtrsim 0.9$ which lie inside the liquid-solid coexistence region (Ref. 20).

DFT, being a mean field theory, is unable to describe correctly the shape of the coexistence curve in the region of the critical point.

Within the present DFT, having calculated the spinodal and binodal for a particular value of $A$, one can obtain results for all other values of $A$ by a simple rescaling of the vertical $\left(\epsilon^{-1}\right)$ axis. It is straightforward to show that, within the DFT, the critical density is independent of $A$. For the case where $A=0$ (not displayed), the present theory yields a critical density within $5 \%$ of the value obtained from the more accurate

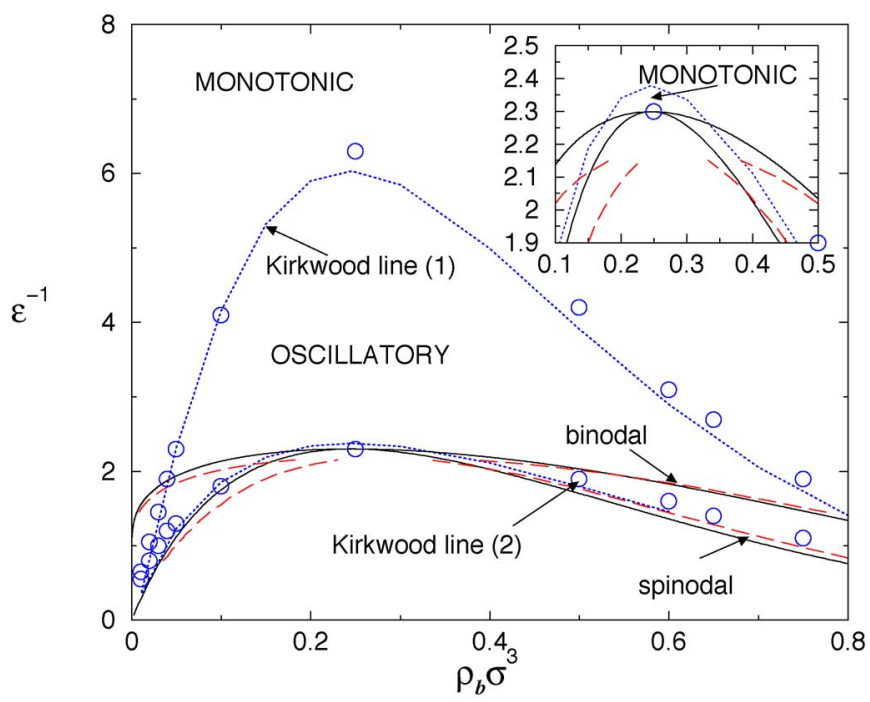

FIG. 4. As in Fig. 3, except here $A=0.02$ and we also display SCOZA results: the dashed lines denote the SCOZA spinodal and binodal and the open circles correspond to points on the two SCOZA Kirkwood lines. Note that within the DFT (RPA) the lower Kirkwood (dotted) line is quite close to the spinodal which means that there is only a very small supercritical region where the asymptotic decay of $h(r)$ is monotonic. A similar scenario pertains to SCOZA (see magnification of the critical region in the inset). 


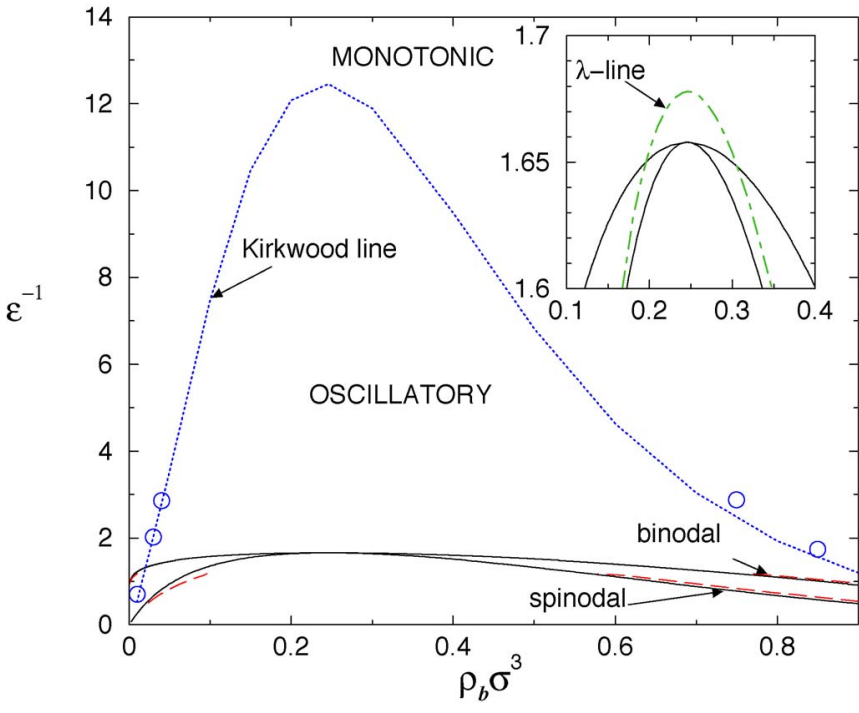

FIG. 5. As in Figs. 3 and 4, except here $A=0.082$. The binodal and spinodal calculated from DFT (solid lines) lie close to the corresponding SCOZA results (dashed lines). Note that solutions to SCOZA do not exist for 1.18 $<\epsilon^{-1}<1.56$ and for $\epsilon^{-1}>4.04$ for this value of $A$ (see text). Within the DFT (RPA) there is a $\lambda$ line (dash-dotted line in magnification in the inset) at which the structure factor $S(k)$ diverges at $k=k_{c} \neq 0$, where $k_{c} \ll 2 \pi / \sigma$.

SCOZA. ${ }^{19}$ However, as $A$ is increased the discrepancy increases. The general trend that the value of $\epsilon^{-1}$ at the critical point is decreased as $A$ is increased is found in both theories. ${ }^{19}$

In Figs. 5 and 6, which refer to $A=0.082$ and 0.5, respectively, the dash dotted line denotes the $\lambda$ line as obtained from the DFT (RPA). The $\lambda$ line takes the shape of a loop which crosses both branches of the binodal and meets the spinodal at values of $\epsilon^{-1}$ below where one would expect the critical point.

When considering the SCOZA results, one should keep in mind that, unlike the RPA, there are regimes in which the theory cannot be solved. In particular, this is the case for values of $A$ and $\epsilon$ such that the competition between attraction and repulsion is very strong, and the fluid is expected to form microphases. In the RPA, this regime is marked by the appearance of the $\lambda$ line, where the structure factor $S(k)$ has a singularity for $k=k_{c} \neq 0$. However, such a singularity is incompatible with the fulfillment of the core condition [Eq. (14)] if the direct correlation function in Fourier space $\hat{c}(k)$ is analytic on the real axis, which is indeed the case in SCOZA. As a consequence, the SCOZA fails to have solutions before a divergence in $S(k)$ occurs. Because of the nonlocal character of the SCOZA PDE, this lack of convergence involves the full range of density. Therefore, it is not possible to locate the $\lambda$ line using SCOZA. Another constraint is related to the numerical integration procedure, which requires the SCOZA PDE to be stable with respect to small fluctuations of the solution. However, such a requirement is not fulfilled when $A$ is sufficiently large that the repulsive tail contribution to the potential overwhelms the attractive one. In this case, the PDE behaves like a diffusion equation with a negative diffusion coefficient and cannot be integrated numerically. An estimate of this intrinsic stability limit is provided by the condition that the spatial integral of the tail

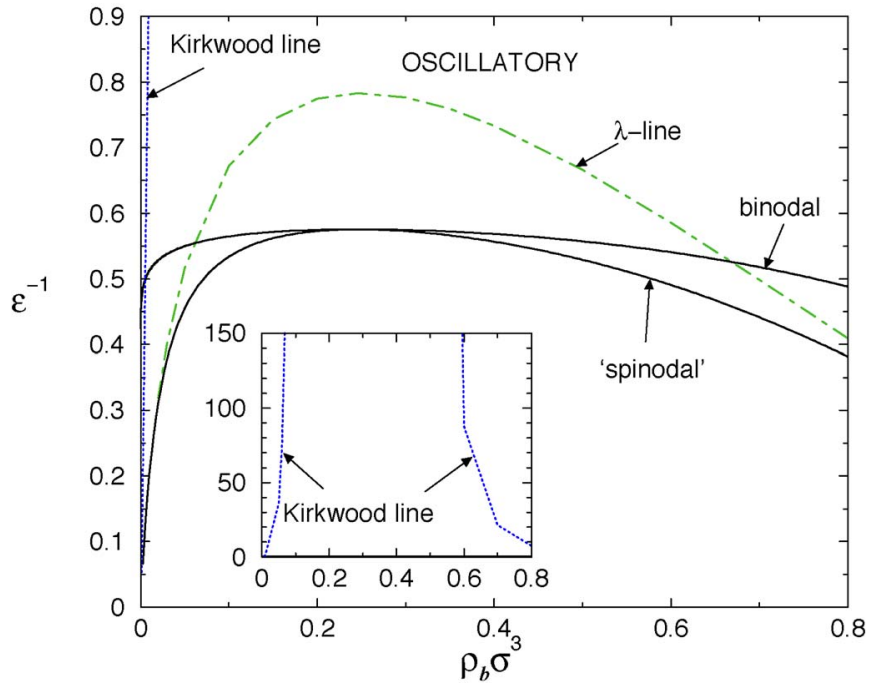

FIG. 6. As in Fig. 3, except here $A=0.5$. Now the region of the phase diagram enclosed by the $\lambda$ line (dash-dotted) is quite large. Furthermore, the region enclosed by the Kirkwood (dotted) line is also very large (see larger scale in the inset). Note that solutions to SCOZA exist only for $\epsilon^{-1}$ $<0.194$ (see text).

potential $4 \pi \int_{\sigma}^{\infty} \mathrm{d} r r^{2} v(r)$ should remain negative. How the above constraints affect the existence of the solution in the present representation, in which the phase diagram is studied as a function of the attraction amplitude $\epsilon$ at fixed repulsion amplitude $A$, can be gleaned by considering the case where $A=0.082$ reported in Fig. 5. For a temperature-independent two-Yukawa potential with the same inverse-range parameters $Z_{1}=1$ and $Z_{2}=0.5$ as those considered here, ${ }^{19}$ the liquidgas transition is found to disappear when the relative amplitude of the repulsion $A / \epsilon$ is larger than 0.097. For $A$ $=0.082$ this requires $\epsilon^{-1}>1.18$. When this condition on $\epsilon$ is met, the theory fails to converge below a certain threshold temperature $T_{\text {th }}$ before liquid-vapor separation takes place. Whether this happens or not in the phase diagram of Fig. 5 depends on whether the reduced temperature $T^{*}=1$ lies below or above the threshold temperature. For $A / \epsilon$ just above 0.097, Fig. 1 of Ref. 19 shows that $T_{\mathrm{th}}^{*} \simeq 1.7 \epsilon$ which, for $\epsilon^{-1} \simeq 1.18$, is indeed above unity. Therefore, the SCOZA solution disappears as soon as $\epsilon^{-1}>1.18$. However, as $\epsilon^{-1}$ increases, the reduced threshold temperature $T_{\text {th }}^{*}$ decreases, until for $\epsilon^{-1}>1.56$ one has $T_{\mathrm{th}}^{*}<1$, so that convergence for $T^{*}=1$ is again achieved. In the RPA picture, this corresponds to being above the maximum of the $\lambda$ line. For larger values of $\epsilon^{-1}$, the SCOZA can be solved. However, the solution disappears again at high values of $\epsilon^{-1}$, when the repulsive part of the interaction dominates. For $Z_{1}=1, Z_{2}=0.5$, and $A$ $=0.082$, the SCOZA PDE fails to converge for $\epsilon^{-1}>4.04$, in fair agreement with the value $\epsilon^{-1}=4.06$ above which the spatial integral of the tail potential for $r>\sigma$ becomes positive. In summary, for $A=0.082$ the SCOZA solution does not exist for $1.18<\epsilon^{-1}<1.56$ and for $\epsilon^{-1}>4.04$. Turning now to the case where $A=0.5$ shown in Fig. 6, the SCOZA solution disappears for $\epsilon^{-1}>0.097 / A=0.194$. However, for this choice of $A$ the solution does not reappear at larger values of $\epsilon^{-1}$ because the region where the competition is strong and a $\lambda$ line is present according to RPA, overlaps with the region 
$\epsilon^{-1}>0.67$ where the integral of the tail potential becomes positive and the SCOZA PDE is unstable. Therefore, for $A$ $=0.5$ only the interval $\epsilon^{-1}<0.194$ is accessible to the SCOZA. We did calculate portions of the binodal and spinodal in this interval, finding good agreement with the DFT results. However, for these comparatively low values of $\epsilon^{-1}$ the relevant densities are either very low or very high, and are not displayed in Fig. 6.

\section{ASYMPTOTIC DECAY OF CORRELATION FUNCTIONS}

In this section we determine the asymptotic decay, $r$ $\rightarrow \infty$, of the total pairwise correlation function $h(r)=g(r)-1$ in our model. The basic procedure follows that in Ref. 27. In Fourier space the OZ relation is given by Eq. (13), or equivalently by

$$
\hat{h}(k)=\frac{\hat{c}(k)}{1-\rho_{b} \hat{c}(k)},
$$

where $\hat{h}(k)$ is the three-dimensional Fourier transform (FT) of $h(r)$. Inverting the FT, and noting that $\hat{h}(k)$ is even, we can write

$$
\begin{aligned}
r h(r) & =\frac{1}{4 \pi^{2} i} \int_{-\infty}^{\infty} \mathrm{d} k k e^{i k r} \hat{h}(k) \\
& =\frac{1}{4 \pi^{2} i} \int_{-\infty}^{\infty} \mathrm{d} k k e^{i k r} \frac{\hat{c}(k)}{1-\rho_{b} \hat{c}(k)},
\end{aligned}
$$

which can be evaluated by contour integration in the complex plane. ${ }^{27}$ We expect the singularities of $\hat{h}(k)$ to be simple poles. Choosing an infinite radius semicircle contour in the upper half of the complex plane, we obtain

$$
r h(r)=\frac{1}{2 \pi} \sum_{n} R_{n} e^{i k_{n} r}
$$

where $R_{n}$ is the residue of $k \hat{h}(k)$ for the $n$th pole at $k=k_{n}$. The $k_{n}$ are solutions of

$$
1-\rho_{b} \hat{c}\left(k_{n}\right)=0 .
$$

In general, there are an infinite number of poles. Poles lying off the imaginary axis occur in conjugate pairs $k_{n}= \pm \alpha_{1}$ $+i \alpha_{0}$ and such a pair contributes a damped oscillatory term of the form $\exp \left(-\alpha_{0} r\right) \cos \left(\alpha_{1} r-\theta\right)$ to the sum in Eq. (18). Poles that lie on the imaginary axis, $k_{n}=i \widetilde{\alpha}_{0}$, contribute a pure exponential term of the form $\exp \left(-\widetilde{\alpha}_{0} r\right)$ to the sum in Eq. (18). The longest range decay of $h(r)$ is determined by the pole(s) with the smallest imaginary part. If $\alpha_{0}<\widetilde{\alpha}_{0}$ the longest range decay is damped oscillatory, but if $\alpha_{0}>\tilde{\alpha}_{0}$, then the asymptotic $r \rightarrow \infty$ decay of $h(r)$ is monotonic.

Given the analytic expression we have for $\hat{c}(k)$ within the DFT (RPA) treatment [Eqs. (9)-(12)], it is fairly straightforward to calculate the full set of solutions $\left\{k_{n}\right\}$ to Eq. (19) for the present model fluid. Thus we are able to determine the pole(s) with the smallest $\alpha_{0}$ and determine the ultimate decay of $h(r)$.

Before presenting our numerical results, we first discuss a simplified model, which should provide insight into the origin of the leading order poles. In Eq. (7), $v_{p}(r)$ takes a constant value for $r<\sigma$. If we replace this constant value by the form that $v(r)$ takes for $r>\sigma$ (i.e., keeping a doubleYukawa form for all values of $r$ ), then the denominator function $D(k) \equiv 1-\rho_{b} \hat{c}(k)$ takes the simple form

$$
D(k)=1-\rho_{b} \hat{c}_{\mathrm{PY}}(k)-\frac{4 \pi \sigma^{3} \epsilon \rho_{b}}{Z_{1}^{2}+q^{2}}+\frac{4 \pi \sigma^{3} A \rho_{b}}{Z_{2}^{2}+q^{2}},
$$

with $q=k \sigma$. We find that this simplified model displays the same pole structure as the full version with $v_{p}(r)$ given by Eq. (7). When $A=0$ this is simplified even further,

$$
D_{0}(k)=1-\rho_{b} \hat{c}_{\mathrm{PY}}(k)-\frac{4 \pi \sigma^{3} \epsilon \rho_{b}}{Z_{1}^{2}+q^{2}} .
$$

We first seek purely imaginary poles and substitute $k=i \widetilde{\alpha}_{0}$ into Eq. (21). Provided $\widetilde{\alpha}_{0} \sigma \lesssim 10$, then $\hat{c}_{\mathrm{PY}}\left(i \widetilde{\alpha}_{0}\right) \approx \hat{c}_{\mathrm{PY}}(0)$, so that

$$
D_{0}(i \widetilde{\alpha}) \approx B-\frac{C}{Z_{1}^{2}-\sigma^{2} \widetilde{\alpha}_{0}^{2}},
$$

where $B \equiv\left[1-\rho_{b} \hat{c}_{\mathrm{PY}}(0)\right]>0$ and $C \equiv 4 \pi \sigma^{3} \epsilon \rho_{b}>0$ are constants dependent on the state point of the fluid. There is only one solution to the equation $D_{0}\left(i \widetilde{\alpha}_{0}\right)=0$, where $D_{0}$ is given by Eq. (22). When $B=C / Z_{1}^{2}$ then we obtain the solution $\widetilde{\alpha}_{0}$ $=0$; this is just the spinodal. Furthermore, the solution is bounded above, i.e., $\tilde{\alpha}_{0} \sigma<Z_{1}$.

We find that there are an infinite number of complex poles. These are essentially just the poles of the hard sphere reference fluid. Of these, the pole with the smallest imaginary part has a real part $\alpha_{1} \approx 2 \pi / \sigma$. The imaginary part of this pole, $\alpha_{0}$, takes relatively large values at low densities and decreases as the density is increased. Thus we find, for the fluid with $A=0$, that at low densities the purely imaginary pole dominates the asymptotic decay of $h(r)$. However, as the density is increased, one finds that at some point $\widetilde{\alpha}_{0}$ $=\alpha_{0}$; on further increasing the density, the asymptotic decay of $h(r)$ crosses over from monotonic to damped oscillatory decay, with a wavelength $\sim \sigma$. The line in the phase diagram defined by the locus $\widetilde{\alpha}_{0}=\alpha_{0}$ is termed the Fisher-Widom (FW) line. ${ }^{27,42}$ For the case when $Z_{1}=1$ and $Z_{2}=0.5$, this line is at high densities. In Fig. 3 we display the FW line for the case $A=0.0001$ where this line is at densities $\rho \sigma^{3}>0.9$ and lies within the liquid-solid coexistence region. ${ }^{20}$

We now consider the case $A \neq 0$. For purely imaginary poles, $k=i \widetilde{\alpha}_{0}$, Eq. (20) becomes [cf. Eq. (22)]

$$
D(i \widetilde{\alpha}) \approx B-\frac{C}{Z_{1}^{2}-\sigma^{2} \widetilde{\alpha}_{0}^{2}}+\frac{E}{Z_{2}^{2}-\sigma^{2} \widetilde{\alpha}_{0}^{2}},
$$

where $E \equiv 4 \pi \sigma^{3} A \rho_{b}>0$ is independent of $\widetilde{\alpha}_{0}$. When $B$ $=C / Z_{1}^{2}-E / Z_{2}^{2}$, then we obtain the solution $\widetilde{\alpha}_{0}=0$ to the equation $D\left(i \widetilde{\alpha}_{0}\right)=0$, which corresponds to the spinodal. When $A$ is sufficiently small that there is no $\lambda$ line in the phase diagram, then we find the following scenario: At the critical point (or more generally, on the spinodal) there are two roots of $D\left(i \widetilde{\alpha}_{0}\right)=0$, the solution $\widetilde{\alpha}_{0}=\widetilde{\alpha}_{0}^{a}=0$ and another at a larger value of $\tilde{\alpha}_{0}=\widetilde{\alpha}_{0}^{b}<Z_{2} / \sigma$. On increasing $\epsilon^{-1}$, moving away from the spinodal, $\alpha_{0}^{a}$ increases in value, while $\alpha_{0}^{b}$ decreases in value. As one moves further from the spinodal these two 
roots (poles) coalesce at a minimum of $D\left(i \widetilde{\alpha}_{0}\right)$ in the interval $0<\widetilde{\alpha}_{0}<Z_{2} / \sigma$. On moving still further from the spinodal, we find that there are no solutions to the equation $D\left(i \widetilde{\alpha}_{0}\right)=0$, i.e., there are no purely imaginary poles in this portion of the phase diagram. Near the spinodal, $D\left(i \widetilde{\alpha}_{0}\right)<0$ for $Z_{2} / \sigma<\widetilde{\alpha}_{0}$ $<Z_{1} / \sigma$. However, on moving away from the spinodal, one finds that in this interval the function increases and its maximum touches the axis $D\left(i \widetilde{\alpha}_{0}\right)=0$. On increasing $\epsilon^{-1}$ further the function crosses the axis and there are two roots (purely imaginary poles) in the interval $Z_{2} / \sigma<\widetilde{\alpha}_{0}<Z_{1} / \sigma$. As one continues to move away from the spinodal these two poles separate, and very far from the spinodal one finds that one pole $\alpha_{0}^{a^{\prime}} \rightarrow Z_{1}^{-} / \sigma$ and the other pole $\alpha_{0}^{b^{\prime}} \rightarrow Z_{2}^{+} / \sigma$.

Analyzing the roots of $D(k)=0$ more generally, we find that when the two purely imaginary poles discussed above coalesce, they form a conjugate pair of complex poles at $k$ $= \pm \alpha_{1}+i \alpha_{0}$. At the point of coalescence $\alpha_{1}=0$, i.e., the wavelength of the oscillations is infinite. For all the relevant values of $\epsilon^{-1}, \alpha_{1}$ remains small, such that $0<\alpha_{1} \ll 2 \pi / \sigma$. It is this conjugate pair of poles which generates the oscillatory decay of $h(r)$ with a wavelength $\gg \sigma$, indicating the tendency toward cluster formation. The line in the phase diagram at which these pairs of purely imaginary poles coalesce is denoted the Kirkwood line. ${ }^{43-45}$ Kirkwood $^{31}$ was the first to describe this mechanism for crossover from monotonic to oscillatory decay in his study of (charge) correlations in electrolytes. Moving away from the spinodal in the phase diagram, the conjugate pair of complex poles with small $\alpha_{1}$ move from the real axis (increasing $\alpha_{0}$ ). Eventually, $\alpha_{1} \rightarrow 0$ and the poles rejoin the imaginary axis at some point in the interval $Z_{2} / \sigma<\widetilde{\alpha}_{0}<Z_{1} / \sigma$, coalescing to form the second pair of purely imaginary poles described above. There is therefore a second Kirkwood line in the phase diagram. In Fig. 7 we display the poles calculated numerically from Eq. (19) with $\hat{c}(k)$ given by Eqs. (9)-(12) along a path at the critical density $\rho \sigma^{3}=0.2457$ for a fluid with $A=0.02, Z_{1}=1$, and $Z_{2}=0.5$. These results exhibit all the features of the simpler model described above.

Figure 7(a) plots the imaginary part of the low lying poles. For small values of $\epsilon^{-1}$ there is a pair of purely imaginary poles [solid line in Fig. 7(a)] coalescing at the first Kirkwood point at $\epsilon^{-1}=2.38$ and evolving as a conjugate complex pair (dash dotted line) until the second Kirkwood point $\epsilon^{-1}=6.03$ when a second pair of pure imaginary poles emerge. Figure 7(b) shows that the real part of the lowest lying complex pole $\alpha_{1} \sigma$ remains $<0.16$ throughout the range where the asymptotic decay of $h(r)$ is oscillatory. We see that the pole with $\alpha_{1} \approx 2 \pi / \sigma$, arising from the reference hard sphere correlations, has $\alpha_{0} \sigma \approx 2.8>Z_{1}$, and therefore for all values of $\epsilon^{-1}$, at this density, this pole does not determine the asymptotic decay of $h(r)$. We also display the next higher order pole arising from the reference hard sphere correlations, which has $\alpha_{1} \approx 4 \pi / \sigma$ and $\alpha_{0} \sigma \approx 4.3$; the imaginary and real parts of these poles are plotted as dashed lines in Figs. 7(a) and 7(b). Note that for the poles originating from the reference hard sphere correlations, the values of $\alpha_{0}$ and $\alpha_{1}$ change very little as $\epsilon^{-1}$ is increased.

As the parameter $A$ is increased, the maximal separation between the two Kirkwood lines increases (see Figs. 3-5);
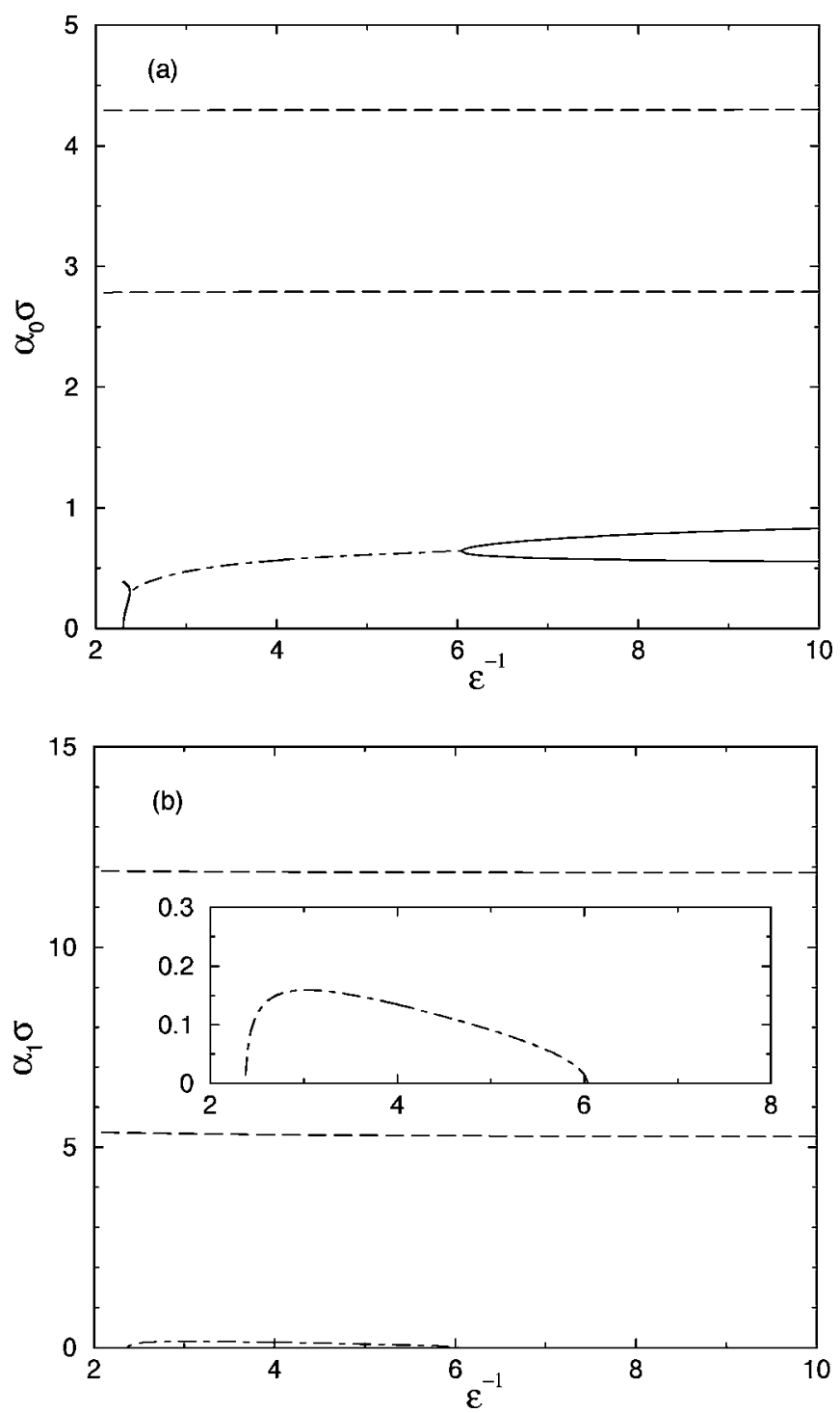

FIG. 7. (a) The imaginary part $\alpha_{0}$ of the low lying poles for a fluid with $A=0.02, Z_{1}=1$, and $Z_{2}=0.5$, calculated within the DFT (RPA) along a line of constant density $\rho \sigma^{3}=0.2457$, the critical density. The dashed lines correspond to complex poles arising from the reference hard sphere correlations, the dot dashed line to a complex pole with a small real part $0<\alpha_{1}$ $\ll 2 \pi / \sigma$, and the solid lines to two purely imaginary poles. The point near $\epsilon^{-1}=2.38$, where the first pair of purely imaginary poles coalesce corresponds to the first Kirkwood point and the point near $\epsilon^{-1}=6.03$ where the second pair coalesce corresponds to the second Kirkwood point for this density (see Fig. 4). (b) The real part $\alpha_{1}$ for the complex poles plotted in (a). The inset is a magnification. Note that $\alpha_{1}=0$ at the two Kirkwood points and remains small in the oscillatory region.

one Kirkwood line moves towards the spinodal (decreasing $\epsilon^{-1}$ ) and the other moves away from the spinodal. Within the DFT the lower Kirkwood line meets the spinodal at a value of $A \simeq 0.051$ (for $Z_{1}=1$ and $Z_{2}=0.5$ ). For greater values of $A$ a $\lambda$ line appears in the phase diagram, enclosing the region where one expects the critical point. The $\lambda$ line corresponds to the situation where the imaginary part $\alpha_{0} \rightarrow 0$ for the complex pole that has a small real component $0<\alpha_{1} \ll 2 \pi / \sigma$ at some point above the spinodal. A divergence is generated in the static structure factor at $k=k_{c}=\alpha_{1}$. Decreasing $\epsilon^{-1}$ and following the $\lambda$ line, one finds that the value of $k_{c}$ decreases continuously and eventually $k_{c} \rightarrow 0$, i.e., the $\lambda$ line converts 
to the spinodal. For the case $Z_{1}=1$ and $Z_{2}=0.5$ we could not obtain a solution for the SCOZA, near where one might expect to find the $\lambda$ line, above a certain value of $A$. On the basis of the discussion at the end of Sec. IV, the value of $A$ above which SCOZA fails to converge is determined by requiring that the threshold value of the relative amplitude ${ }^{19}$ $A / \epsilon=0.097$ is reached when the corresponding threshold temperature $T_{\mathrm{th}}^{*} \simeq 1.7 \epsilon$ is equal to unity. This gives $A$ $\gtrsim 0.057$. This bound on $A$ is somewhat larger than the value $A=0.051$ predicted by the present DFT.

We confirmed that the scenarios for the pole structure described in Fig. 7 are also present in the SCOZA theory. The calculation of poles is straightforward because in SCOZA one has analytic expressions for $\hat{c}(k) .{ }^{40}$ In Figs. 4 and 5 we display the Kirkwood lines (open circles) calculated from the SCOZA alongside those from DFT (dotted lines). We find good agreement between the results, demonstrating that our simple DFT (RPA) describes correctly the behavior of the poles in the model fluid.

We emphasize that there are two distinct mechanisms for a crossover in the asymptotic decay of $h(r)$ from monotonic to damped oscillatory: (i) the coalescence of two purely imaginary poles to form a conjugate pair of complex poles defines a point on the Kirkwood line and (ii) the simpler FW mechanism where, on decreasing the fluid density, a purely imaginary pole descends down the imaginary axis and acquires an imaginary part $\widetilde{\alpha}_{0}$ smaller than that of the dominant complex pole with imaginary part $\alpha_{0}$, so that the ultimate decay becomes monotonic. As mentioned earlier, when $Z_{1}$ $=1$ and $Z_{2}=0.5$, the FW line is at high densities, inside the region where we expect the solid phase to be the equilibrium state (see Fig. 3). However, for larger values of $Z_{1}$ and $Z_{2}$ we find that the FW line moves to lower densities, where it can intersect the Kirkwood lines. In Fig. 8 we display the phase diagram for a fluid with $Z_{1}=6, Z_{2}=2$, and $A=0.3$. We find that when the Kirkwood and FW lines intersect, another kind of structural crossover line appears in the phase diagram: there is a crossover from damped oscillatory decay with one wavelength to damped oscillatory decay with another wavelength. ${ }^{46,47}$ In the present case the crossover is from a decay with a long wavelength of many particle diameters (clustering in the fluid) to a decay with a wavelength $\sim \sigma$ as $\epsilon^{-1}$ is increased (see Figs. 8 and 9). Note that for the choice of parameters corresponding to the phase diagram in Fig. 8, the liquid-gas transition may be weakly metastable with respect to the fluid-solid transition. However, this does not affect the crossover behavior of correlations, which is in a region of the phase diagram far from any phase transitions. ${ }^{20}$

The pole analysis described above points to those regions of the phase diagram where clustering might occur, i.e., the region between the Kirkwood lines. However, so far we have determined only the form of the asymptotic decay of $h(r):$ whether it is monotonic or oscillatory as $r \rightarrow \infty$. One must also calculate the amplitude of the longest wavelength oscillatory contribution to $h(r)$ in order to assess how significant clustering is in the fluid. This is straightforward within the present DFT. The amplitude is determined by the residue of the pole [see Eq. (18)], which is given by

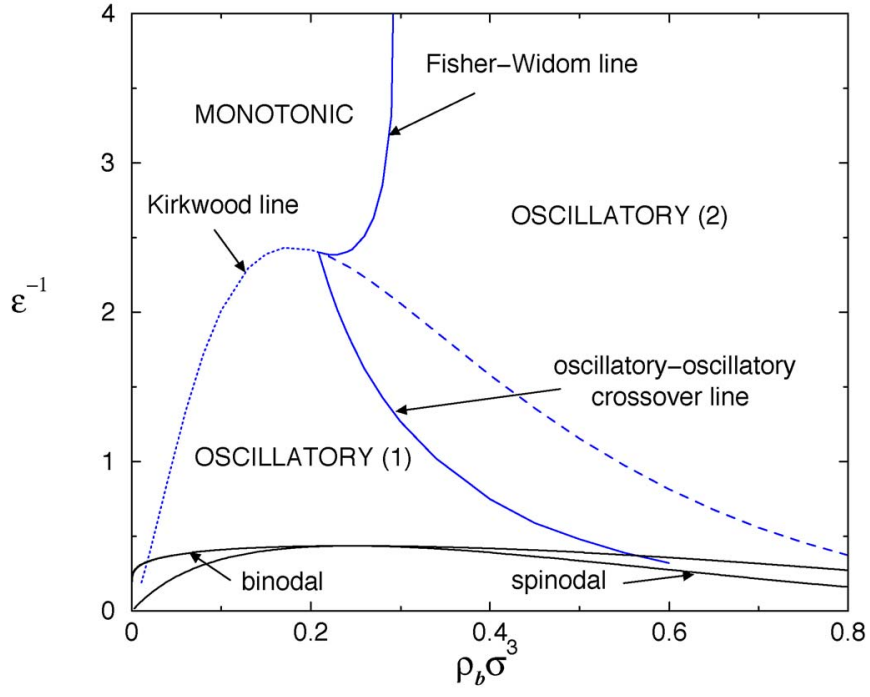

FIG. 8. Phase diagram for the case when $Z_{1}=6, Z_{2}=2$, and $A=0.3$. The Kirkwood line (dotted) denotes the line at which the asymptotic decay of $h(r)$ crosses over from monotonic to damped oscillatory with wavelength $\gg \sigma$, and the FW line the crossover from monotonic to damped oscillatory with wavelength $\sim \sigma$. Between the two regions of oscillatory decay there is another (oscillatory to oscillatory) crossover line. The dashed line is the continuation of the Kirkwood line, although in this region of the phase diagram it denotes crossover between higher order poles, i.e., those with larger values of $\alpha_{0}$ than the leading order pole. There is also a second Kirkwood line located just above the critical point so that in the immediate vicinity of the critical point $h(r)$ decays monotonically (OZ-type). However, on the scale of this figure, this second Kirkwood line is indistinguishable from the spinodal.

$$
R_{n}=\frac{-k_{n} \hat{c}\left(k_{n}\right)}{\rho_{b} \hat{c}^{\prime}\left(k_{n}\right)},
$$

where the prime denotes the derivative with respect to $k$. For states just below the upper Kirkwood line the amplitude is generally quite small. For example, when $A=0.02$, for the fluid with $Z_{1}=1$ and $Z_{2}=0.5$ (see phase diagram in Fig. 4), at the state point with $\rho_{b} \sigma^{3}=0.25$ and $\epsilon^{-1}=5$, there is a complex pole with $\alpha_{1} \sigma=0.0909$ and $\alpha_{0} \sigma=0.613$. This makes a damped oscillatory contribution to $h(r)$ of the form $\mathcal{A} \exp \left(-\alpha_{0} r\right) \cos \left(\alpha_{1} r-\theta\right) / r$, with amplitude $\mathcal{A}=0.0994$ and phase $\theta=0.992$; i.e., this term gives quite a small contribution to $h(r)$, despite being the term that determines the ultimate asymptotic decay. However, further below the Kirkwood line the amplitude $\mathcal{A}$ can become larger and in the vicinity of a $\lambda$ line, $\mathcal{A}$ can be very large so that the pole provides a large contribution to $g(r)=1+h(r)$ (see, for example, the results in Fig. 2). Indeed, when the $\lambda$ line is reached, the amplitude $\mathcal{A}$ diverges since the pole $k_{n}$ in Eq. (24) corresponds to a maximum of $\hat{c}(k)$ on the real axis and $\hat{c}^{\prime}\left(k_{n}\right)$ vanishes. We will return to this point in Sec. VII.

\section{INHOMOGENEOUS FLUID: DENSITY PROFILES AT INTERFACES}

In this section we turn our attention away from the uniform fluid to situations where the one-body density is spatially varying. We consider two separate cases: (a) the fluid adsorbed at a hard wall and (b) the liquid-gas interface. In both cases the pole analysis for the decay of $h(r)$ is directly 

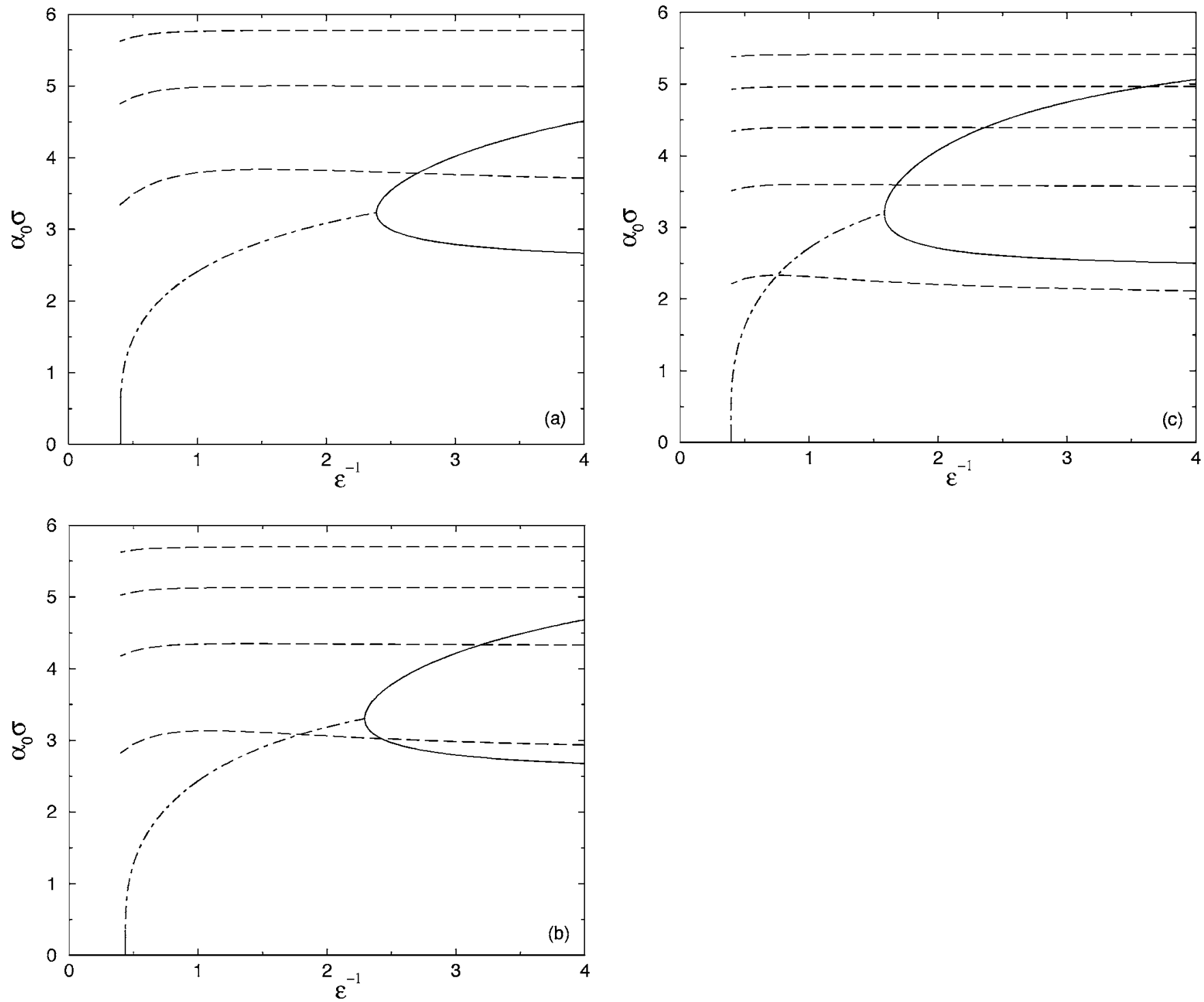

FIG. 9. The imaginary part $\alpha_{0}$ of the lowest lying poles for a fluid with $Z_{1}=6, Z_{2}=2$, and $A=0.3$, calculated within the DFT (RPA) along lines of constant density: (a) $\rho \sigma^{3}=0.15$, (b) the critical density $\rho \sigma^{3}=0.2457$, and (c) $\rho \sigma^{3}=0.4$. The dashed lines correspond to complex poles arising from the reference hard sphere correlations, the dot dashed line to the complex pole with a small real part $0<\alpha_{1} \ll 2 \pi / \sigma$, and the two solid lines to two purely imaginary poles. The point where the two lowest lying pure imaginary poles coalesce corresponds to a Kirkwood point [see (a)]. In (b) this coalescence occurs between higher order poles. The crossover at $\epsilon^{-1}=1.79$ is between complex poles with different oscillatory wavelengths (see the line in Fig. 8). The subsequent crossover at $\epsilon^{-1}$ $=2.42$ is from oscillatory to monotonic decay. In (c) there is oscillatory to oscillatory crossover at $\epsilon^{-1}=0.75$. The pole with $\alpha_{1} \sigma \sim 2 \pi$ remains the dominant one for larger $\epsilon^{-1}$. Note that in (a)-(c) there is a second Kirkwood point at $\epsilon^{-1} \approx 0.4$, very near the critical value of $\epsilon^{-1}$, so that at smaller values of $\epsilon^{-1}$ (close to the spinodal) there are two purely imaginary poles, similar to the results displayed in Fig. 7(a).

relevant to the decay of inhomogeneous fluid one-body density profiles; the same pole(s) which determine the asymptotic decay of $h(r)$ determine the asymptotic decay of the one-body density profiles. ${ }^{27}$ For density profiles which vary only in one (Cartesian) direction, e.g., when the external potential $V_{\text {ext }}(\mathbf{r}) \equiv V_{\text {ext }}(z)$, then provided $V_{\text {ext }}(z)$ is sufficiently short ranged the longest range decay of the profile into bulk at $z=\infty$ takes the form

$$
\rho(z)-\rho_{b} \sim \rho_{b} \mathcal{A}_{w} \exp \left(-\tilde{\alpha}_{0} z\right), \quad z \rightarrow \infty
$$

when the pole with the smallest value of $\alpha_{0}$ is purely imaginary and

$$
\rho(z)-\rho_{b} \sim \rho_{b} \widetilde{\mathcal{A}}_{w} \exp \left(-\alpha_{0} z\right) \cos \left(\alpha_{1} z-\theta_{w}\right), \quad z \rightarrow \infty
$$

when the pole with the smallest $\alpha_{0}$ is complex. The poles $k_{n}=i \widetilde{\alpha}_{0}$ or $k_{n}= \pm \alpha_{1}+i \alpha_{0}$ correspond to the bulk density $\rho_{b}{ }^{27}$

Using the DFT described in Sec. II, we have determined the one-body density profiles $\rho(z)$ for the two cases mentioned above. Minimizing our approximation for $\Omega_{V}[\rho]$ yields an Euler-Lagrange equation for the equilibrium density profile $\rho(z)$ which can be solved by means of a standard iterative scheme. In Fig. 10, we display the one-body density profiles at a planar hard wall with 


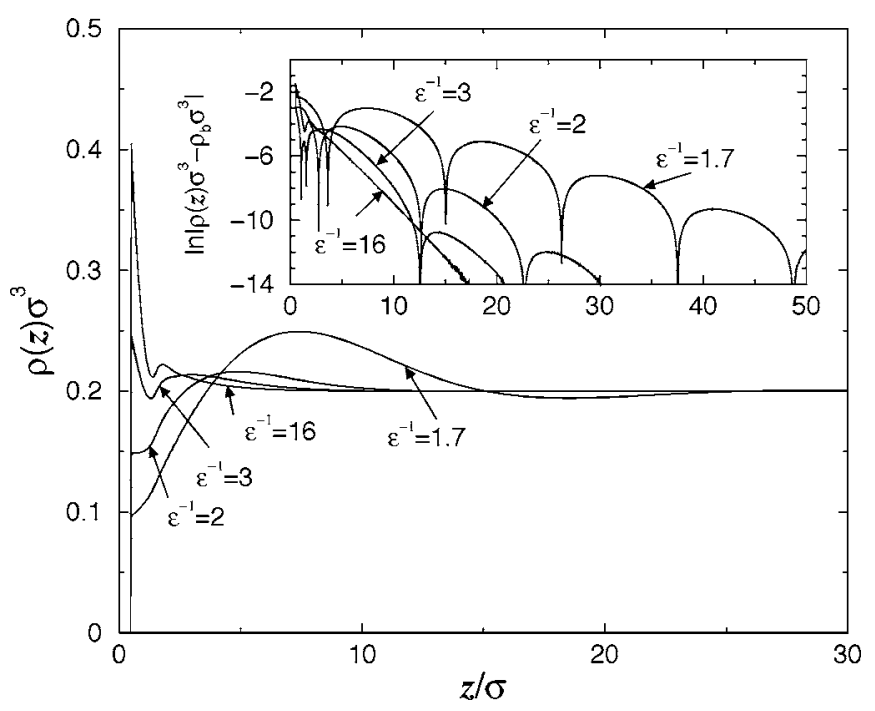

FIG. 10. Density profiles $\rho(z)$ at a planar hard wall for a fluid with parameters $A=0.082, Z_{1}=1$, and $Z_{2}=0.5$ (see the phase diagram in Fig. 5). The profiles are calculated for a fixed bulk density $\rho_{b} \sigma^{3}=0.2$, for $\epsilon^{-1}=1.7,2,3$, and 16. In the inset we plot $\ln \left|\rho(z) \sigma^{3}-\rho_{b} \sigma^{3}\right|$. For $\epsilon^{-1}=16$ the asymptotic decay of $\rho(z)$ is monotonic (exponential). The other three states lie on the oscillatory side of the Kirkwood line. Note that the decay length increases as $\epsilon^{-1}$ is reduced and the $\lambda$ line is approached. For a given value of $\epsilon^{-1}$ the wavelength of the oscillations is the same as that of $g(r)$ in Fig. 2.

$$
V_{\text {ext }}(z)= \begin{cases}\infty, & z<\sigma / 2 \\ 0, & z>\sigma / 2 .\end{cases}
$$

The fluid has parameters $A=0.082, Z_{1}=1$, and $Z_{2}=0.5$ (see phase diagram in Fig. 5). The density profiles are calculated for a fixed bulk density $\rho_{b} \sigma^{3}=0.2$, for $\epsilon^{-1}=16,3,2$, and 1.7. For $\epsilon^{-1}=16$ the state point is above the Kirkwood line, and the bulk asymptotic decay of $h(r)$ is monotonic of the form in Eq. (25). This is confirmed in the inset of Fig. 10 which plots $\ln \left|\rho(z) \sigma^{3}-\rho_{b} \sigma^{3}\right|$ vs $z$; when the decay of the density profile is of the form in Eq. (25) the decay of the profile in this representation is a straight line. The other profiles are calculated for state points inside the Kirkwood line, with increasing proximity to the $\lambda$ line. From the inset to Fig. 10 we see that the decay of these profiles is indeed of the form in Eq. (26). The profile for $\epsilon^{-1}=1.7$, a state point near the $\lambda$ line, has a long decay length and oscillates with a long wavelength of about $23 \sigma$. The density profiles for state points in the vicinity of the $\lambda$ line are significantly different from those far away. These profiles should be compared with the radial distribution functions $g(r)$ in Fig. 2 where the same trends with $\epsilon^{-1}$ are observed.

It is striking that as $\epsilon^{-1}$ is reduced the contact density $\rho(\sigma / 2)$ reduces dramatically and for $\epsilon^{-1}=1.7$ there is a region where the density near the wall is significantly depleted below the bulk value out to distances of about $4 \sigma$. That the density at contact should become smaller follows from the hard-wall sum rule: $k_{B} T \rho(\sigma / 2)=p$, where $p$ is the pressure of the bulk fluid far from the wall. Decreasing $\epsilon^{-1}$ corresponds to turning on more of the attractive interaction, thereby decreasing $p$.

In Fig. 11 we display the density profiles for the free planar gas-liquid interface of the same fluid. Profiles are calculated for coexisting states with $\epsilon^{-1}=1.4,1.5,1.6$, and 1.64.

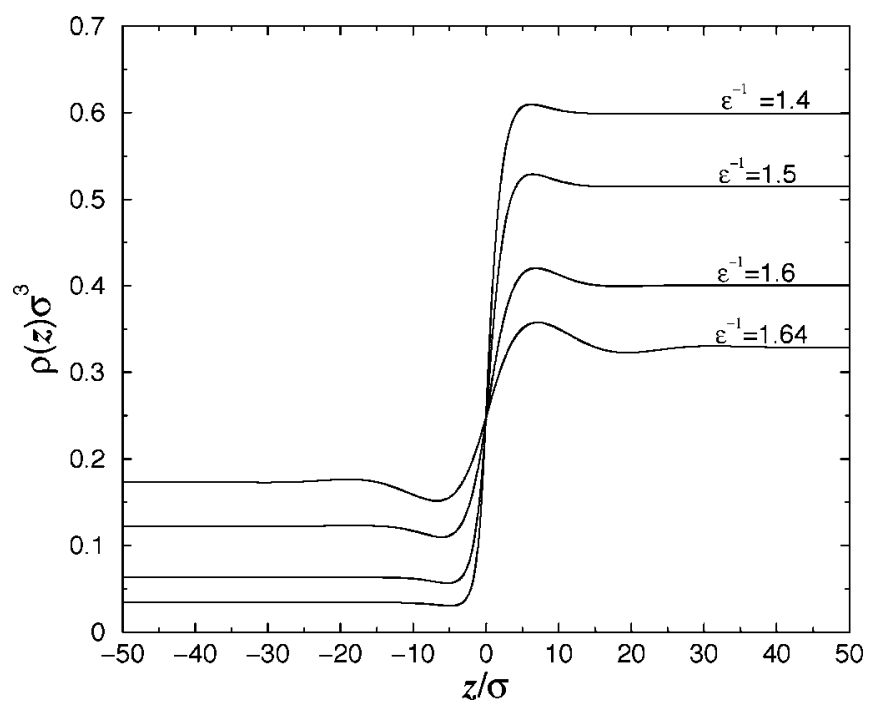

FIG. 11. Density profiles for the free gas-liquid interface calculated for various values of $\epsilon^{-1}$, for a fluid with parameters $A=0.082, Z_{1}=1$, and $Z_{2}=0.5$ (see the phase diagram in Fig. 5). This fluid has a $\lambda$ line close to where one expects the critical point. We find that as $\epsilon^{-1}$ is increased, the density difference between the coexisting phases decreases and the profiles become more structured, i.e., the oscillations become more pronounced on both sides of the interface. This is opposite to what occurs in simple fluids and reflects the proximity of the coexisting states to the $\lambda$ line.

This fluid exhibits a $\lambda$ line enclosing the region of the phase diagram where one expects the critical point, see the inset to Fig. 5. States outside the $\lambda$ line, i.e., for $\epsilon^{-1} \lesssim 1.65$, correspond to conventional coexistence between (disordered) liquid and gas. However, as the four coexisting states that we consider lie inside the Kirkwood line, within our mean field DFT we expect the asymptotic decay of the density profiles to be damped oscillatory on both sides of the interface. This is indeed what we find. Such behavior was found previously in DFT studies of fluid-fluid interfaces of the binary Gaussian core model. ${ }^{46}$ What is striking about the present results and what makes them different from results for other models is that as $\epsilon^{-1}$ is increased and the density difference between the coexisting phases decreases, the profiles become more structured at the interface. Normally, the interfacial density profile becomes less structured when the difference in density between the coexisting phases decreases. The difference is due to the fact that the present system exhibits a $\lambda$ line and the coexisting densities become closer to this line as $\epsilon^{-1}$ is increased (see Fig. 5). The proximity of the $\lambda$ line implies long wavelength oscillatory density profiles decaying slowly into bulk on either side of the interface. It should also imply that the propensity towards cluster formation in both phases is stronger. Clustering should manifest itself in highly structured interfacial profiles.

\section{DISCUSSION}

Using a simple DFT and the SCOZA integral equation theory, we have investigated the bulk structure and phase behavior of a model colloidal fluid with competing interactions. In particular, we have examined in detail the asymptotic decay of the radial distribution function $g(r)$ and have found a rich variety of decay scenarios. The presence of 
clustering in the fluid, which occurs for sufficiently large values of the repulsion amplitude $A$ in the pair potential [Eq. (1)], is reflected in a long wavelength $(\gg \sigma)$ damped oscillatory decay of $g(r)$. For small values of $A$ the region of the phase diagram in which the decay of $g(r)$ is long wavelength oscillatory is bounded above and below by two Kirkwood lines (see Figs. 3 and 4). On these lines the asymptotic decay of $g(r)$ crosses over from damped oscillatory to monotonic (exponential) decay. As $A$ is increased we find within the mean field DFT (RPA) that the lower Kirkwood line is replaced by a $\lambda$ line (see Figs. 5 and 6), indicating a transition to a phase with undamped periodic density fluctuations, e.g., a cluster or a stripe phase. Note that the appearance of a region in the phase diagram with long wavelength oscillatory decay of correlation functions is not restricted to fluids with competing interactions. Such behavior is to be expected in any system with competing interactions. ${ }^{48,49}$ In Ref. 49 the authors investigate the form of the correlation functions in frustrated $O(n)$ spin systems. They find that as the strength of the frustration parameter (the analog of the parameter $A$ in the present system) is increased, a region in the phase diagram opens up in which the decay of spin-spin correlations is oscillatory, crossing over above and below to regions of monotonic decay, in much the same way as in the present fluid system.

In general, the results of our simple mean field DFT (RPA) are in good qualitative and sometimes quantitative agreement with the more sophisticated (and more accurate) SCOZA theory. This reflects the fact that our focus has been on state points where the parameters $A, \epsilon \lesssim 1$. Recall that $(-\epsilon+A) k_{B} T$ is the value of the pair potential at contact, $r=\sigma^{+}$, so that we have focused on cases where the portion of the pair potential that we treat in mean field fashion has an amplitude $\lesssim k_{B} T$. We expect the present mean field DFT to be less reliable when $(\epsilon-A)>1$, or when the width of the attractive portion of the pair potential becomes narrow, i.e., when $Z_{1}$ becomes very large. In the present study we have, in the main, avoided these regimes; an exception is the system described in Fig. 8 where $Z_{1}=6$.

In Sec. VI we presented results from DFT for inhomogeneous one-body fluid density profiles. The oscillatory density profiles in Figs. 10 and 11 correspond to state points between the Kirkwood and the $\lambda$ line in the phase diagram of Fig. 5, where the system is a disordered fluid, albeit with a propensity towards cluster formation. For state points in the vicinity of the $\lambda$ line we find some very striking density profiles with pronounced long wavelength $(\gg \sigma)$ oscillations. It is important to inquire whether such behavior pertains beyond the mean field (MF) treatment we present here.

Determining the existence or nonexistence of a $\lambda$ line is a problem that arises for other types of fluids, e.g., microemulsions near the transition to a lamellar phase and smectic liquid crystals. Recently, much effort has been focused on a possible $\lambda$ transition for charge ordering in the restricted primitive model (RPM) in which the two species of ions are modeled by equal sized hard spheres carrying equal and opposite charges. ${ }^{30}$ It is well known that in the RPM the location of the $\lambda$ line in the phase diagram depends sensitively on the choice of (MF) approximation (see Ref. 30 and refer- ences therein). For example, a simple DFT yields a $\lambda$ line of continuous transitions between a uniform disordered phase and a charge-ordered phase, whereas the more sophisticated MSA predicts no $\lambda$ transition. For the former case Ciach et $a l^{30}$ studied correlation functions in some detail and found that on approaching the $\lambda$ line, from the disordered side, both the decay length and the amplitude of the charge-charge pair correlation function diverge as $\tau^{-1 / 2}$, where $\tau$ measures the "distance" of the state point from the $\lambda$ line. The derivation of Ciach et al. is based on a particular form for the (chargecharge) inverse correlation function. However, their results generalize straightforwardly ${ }^{50}$ to any fluid system where the dominant poles approach the real axis at $\operatorname{Re}\left(k_{n}\right)=k_{c} \neq 0$ and the other poles are well removed. As this situation pertains in the present DFT (RPA) treatment of our model fluid, we expect that close to the $\lambda$ transition,

$$
r h(r) \sim \mathcal{A} \exp \left(-\alpha_{0} r\right) \sin \left(k_{c} r\right), \quad r \rightarrow \infty,
$$

with $\alpha_{0} \sim \tau^{1 / 2}$ and $\mathcal{A} \sim \tau^{-1 / 2}$, where again $\tau$ measures the distance from the $\lambda$ line. This result arises also in a simplified treatment based on the small- $k$ expansion of the direct correlation function: $1 / S(k)=1-\rho \hat{c}(k) \sim a+b k^{2}+c k^{4}$. For $a>0$, $c>0$, the condition that $1 / S(k)$ should be positive definite for every $k>0$ is violated for $b<0, b^{2}-4 a c \geqslant 0$, and the appearance of the $\lambda$ line corresponds to the marginal case where $b^{2}-4 a c=0$. As the $\lambda$ line is approached from the disordered side, one has $b<0, b^{2}-4 a c \rightarrow 0^{-}$, which via contour integration, gives Eq. (28) with $\mathcal{A} \sim 1 / \sqrt{4 a c-b^{2}}$. Clearly the MF treatment implies the unphysical result that pair correlations are unbounded on approaching the $\lambda$ line. What occurs beyond the MF theory? For the RPM a field theoretic treatment, ${ }^{51}$ based on the approach of Brazovskii, ${ }^{52}$ shows that incorporating fluctuations leads to the disappearance of the $\lambda$ transition. Rather, a first-order transition to a chargeordered (crystalline) phase occurs at a temperature below that of the original MF $\lambda$ transition. (Note that the $\lambda$ transition is absent in simulation studies of the continuum RPM.) Moreover, the charge-charge correlation function changes smoothly near the original MF $\lambda$ transition; the decay length and amplitude vary continuously. ${ }^{53}$

One might infer that the very striking long-ranged decay of $g(r)$ observed in Fig. 2 for $\epsilon^{-1}=1.66$, at a state point rather close to the $\lambda$ line in Fig. 5, is an artifact of the MF treatment since including fluctuation effects would remove the $\lambda$ transition, replacing this with a first-order transition to some ordered state (cluster or stripe phase, perhaps). For state points somewhat further from the $\lambda$ line, such as $\epsilon^{-1}=2.0$, the long wavelength oscillations still develop in $g(r)$, but these are more highly damped. Nevertheless, the corresponding structure factor $S(k)$ exhibits a pronounced peak at a nonzero wave number $k_{c}$ (see Fig. 1). The fact that SCOZA yields a very similar structure factor gives us some confidence in the results of the mean field DFT (RPA) for such state points. On the other hand, unlike the RPA, SCOZA does not yield a $\lambda$ line. Rather, it fails to converge in the regime in which a $\lambda$ line is expected according to the RPA. As the convergence limit is approached, the cluster peak in $S(k)$ may reach values much larger than unity, but nevertheless the peak height does not grow arbitrarily, since a singularity in $S(k)$ at nonvanish- 
ing $k$ is incompatible with the core condition [Eq. (14)], which is fulfilled in SCOZA. This can be regarded as an indication that the $\lambda$ transition will be removed when one goes beyond the MF approximation. It is tempting to introduce a rough and ready criterion, analogous to the Hansen-Verlet criterion for freezing, that establishes when we expect the MF treatment to fail or to imply the onset of ordering. Such a criterion might be when the peak height $S\left(k_{c}\right) \sim \mathcal{O}\left(10^{1}\right)$.

Returning to the one-body profiles, we note that none of the bulk state points in Fig. 10 for adsorption at the planar hard wall are particularly close to the $\lambda$ line, and we might expect our DFT results to be at least qualitatively correct. However, in Fig. 11 for the density profiles at the planar liquid-gas interface it is clear that the striking increase in structuring as $\epsilon^{-1}$ is increased is a direct consequence of the close proximity of the $\lambda$ line. It is unlikely that such behavior could survive beyond MF. A possible scenario when fluctuations are included is the phase diagram of Fig. 4 where there are two Kirkwood lines and the critical point lies below the lower line [labeled (2) in Fig. 4]. The MF DFT would yield monotonically decaying profiles on both sides of the interface for coexisting states just below the critical point, but for $1.9 \lessgtr \epsilon^{-1} \lesssim 2.25$ one would expect damped oscillatory profiles on both the liquid and the gas side. For states with $\epsilon^{-1} \lesssim 1.9$, where the upper Kirkwood line [labeled (1) in Fig. 4] intersects the gas binodal, the asymptotic decay on the gas side would be monotonic. Of course, the MF DFT omits effects of capillary wavelike fluctuations that act to erode the amplitude of the oscillations; this mechanism is discussed in Ref. 26 for the case of model colloid-polymer mixtures.

We believe that much more work is required to understand the nature of the fluid correlations in such systems, particularly in regions of the phase diagram where the present MF theory predicts a $\lambda$ line. Indeed, some of us are already engaged in such studies using Monte Carlo simulations and various integral equation theories. Of course, one could also employ DFT to investigate possible spontaneous ordering, i.e., whether there are solutions corresponding to nonuniform (e.g., periodic; stripe or cluster) phases that have a lower free energy than those corresponding to a uniform phase.

We conclude by speculating that since colloidal systems with competing interactions display modulated structures, these systems will have interesting optical properties and may have applications in display technologies.

\section{ACKNOWLEDGMENTS}

One of the authors (A.J.A.) is grateful for the support of EPSRC under Grant No. GR/S28631/01. Two of the authors (D.P and L.R.) acknowledge support from the Marie Curie program of the European Union, Contract No. MRTNCT2003-504712. The authors have benefited from illuminating correspondence with Professor Alina Ciach on the subject of $\lambda$ transitions and the failings of mean field treatments, and they are grateful to Dr. Nigel Wilding for several helpful discussions.
${ }^{1}$ F. Ghezzi and J. C. Earnshaw, J. Phys.: Condens. Matter 9, L517 (1997).

${ }^{2}$ R. P. Sear, S.-W. Chung, G. Markovich, W. M. Gelbart, and J. R. Heath, Phys. Rev. E 59, R6255 (1999).

${ }^{3}$ A. Stradner, H. Sedgwick, F. Cardinaux, W. C. K. Poon, S. U. Egelhaaf, and P. Schurtenberger, Nature (London) 432, 492 (2004).

${ }^{4}$ H. Sedgwick, S. U. Egelhaaf, and W. C. K. Poon, J. Phys.: Condens. Matter 16, S4913 (2004)

${ }^{5}$ A. I. Campbell, V. J. Anderson, J. S. van Duijneveldt, and P. Bartlett, Phys. Rev. Lett. 94, 208301 (2005).

${ }^{6}$ R. Sanchez and P. Bartlett, J. Phys.: Condens. Matter 17, S3551 (2005). ${ }^{7}$ J.-L. Barrat and J.-P. Hansen, Basic Concepts for Simple and Complex Liquids (Cambridge University Press, Cambridge, 2003).

${ }^{8}$ Note that depending on solvent salt concentrations and the polymer radius of gyration one can find cases where the depletion attraction can be longer ranged than the screened Coulombic repulsion. For a discussion of colloid-polymer mixtures in this limit see A. Fortini, M. Dijkstra, and R. Tuinier, J. Phys.: Condens. Matter 17, 7783 (2005) and references therein.

${ }^{9}$ D. Andelman, F. Brochard, and J.-F. Joanny, J. Chem. Phys. 86, 3673 (1986).

${ }^{10}$ G. F. Kendrick, T. J. Sluckin, and M. J. Grimson, Europhys. Lett. 6, 567 (1988).

${ }^{11}$ R. P. Sear and W. M. Gelbart, J. Chem. Phys. 110, 4582 (1999).

${ }^{12}$ J. Groenewold and W. K. Kegel, J. Phys. Chem. B 105, 11702 (2001).

${ }^{13}$ J. Groenewold and W. K. Kegel, J. Phys.: Condens. Matter 16, S4877 (2004).

${ }^{14}$ A. Imperio and L. Reatto, J. Phys.: Condens. Matter 16, S3769 (2004).

${ }^{15}$ F. Sciortino, S. Mossa, E. Zaccarelli, and P. Tartaglia, Phys. Rev. Lett. 93, 055701 (2004).

${ }^{16}$ S. Mossa, F. Sciortino, P. Tartaglia, and E. Zaccarelli, Langmuir 20, 10756 (2004).

${ }^{17}$ F. Sciortino, P. Tartaglia, and E. Zaccarelli, J. Phys. Chem. B 109, 21942 (2005).

${ }^{18}$ A. Imperio and L. Reatto, J. Chem. Phys. 124, 164712 (2006).

${ }^{19}$ D. Pini, G. Jialin, A. Parola, and L. Reatto, Chem. Phys. Lett. 372, 209 (2000).

${ }^{20}$ D. Pini, A. Parola, and L. Reatto, J. Phys.: Condens. Matter 18, S2305 (2006).

${ }^{21}$ J. Wu, Y. Liu, W.-R. Chen, J. Cao, and S.-H. Chen, Phys. Rev. E 70, 050401 (2004).

${ }^{22}$ Y. Liu, W.-R. Chen, and S.-H. Chen, J. Chem. Phys. 122, 044507 (2005).

${ }^{23}$ M. Broccio, D. Costa, Y. Liu, and S.-H. Chen, J. Chem. Phys. 124, 084501 (2006).

${ }^{24}$ S. Asakura and F. Oosawa, J. Chem. Phys. 22, 1255 (1954); J. Polym. Sci. 33, 183 (1958)

${ }^{25}$ A. Vrij, Pure Appl. Chem. 48, 471 (1976).

${ }^{26}$ For a recent survey see J. M. Brader, R. Evans, and M. Schmidt, Mol. Phys. 101, 3349 (2003).

${ }^{27}$ R. Evans, R. J. F. Leote de Carvalho, J. R. Henderson, and D. C. Hoyle, J. Chem. Phys. 100, 591 (1994).

${ }^{28}$ J. S. Høye and G. Stell, Mol. Phys. 52, 1071 (1984); , Int. J. Thermophys. 6, 561 (1985); D. Pini, J. S. Høye, and G. Stell, ibid. 19, 1029 (1998)

${ }^{29}$ D. Pini, G. Stell, and N. B. Wilding, Mol. Phys. 95, 483 (1998).

${ }^{30}$ We borrow the terminology from studies of ionic fluids. see, e.g., G. Stell, J. Stat. Phys. 78, 197 (1995) or A. Ciach, W. T. Góźdź, and R. Evans, J. Chem. Phys. 118, 3702 (2003).

${ }^{31}$ J. G. Kirkwood, Chem. Rev. (Washington, D.C.) 19, 275 (1936).

${ }^{32}$ A. J. Archer, C. N. Likos, and R. Evans, J. Phys.: Condens. Matter 16, L297 (2004).

${ }^{33}$ K. Kroy, M. E. Cates, and W. C. K. Poon, Phys. Rev. Lett. 92, 148302 (2004).

${ }^{34}$ See, e. g., R. Evans, in Fundamentals of Inhomogeneous Fluids, edited by D. Henderson (Dekker, New York), (1992), Chap. 3.

${ }^{35}$ Y. Rosenfeld, Phys. Rev. Lett. 63, 980 (1989).

${ }^{36}$ Y. Rosenfeld, D. Levesque, and J.-J. Weis, J. Chem. Phys. 92, 6818 (1990).

${ }^{37}$ Y. Rosenfeld, J. Chem. Phys. 93, 4305 (1990).

${ }^{38}$ N. W. Ashcroft and J. Lekner, Phys. Rev. 145, 83 (1966).

${ }^{39}$ J.-P. Hansen and I. R. McDonald, Theory of Simple Liquids, 2nd ed. (Academic, London, 1986)

${ }^{40}$ J. S. Høye, G. Stell, and E. Waisman, Mol. Phys. 32, 209 (1976); J. S. Høye and G. Stell, ibid. 52, 1057 (1984); 52, 1071 (1984). 
${ }^{41}$ D. Pini, G. Stell, and N. B. Wilding, J. Chem. Phys. 115, 2702 (2001).

${ }^{42}$ M. E. Fisher and B. Widom, J. Chem. Phys. 50, 3756 (1969).

${ }^{43}$ R. J. F. Leote de Carvalho and R. Evans, Mol. Phys. 83, 619 (1994).

${ }^{44}$ R. J. F. Leote de Carvalho and R. Evans, Mol. Phys. 92, 211 (1997).

${ }^{45}$ P. Hopkins, A. J. Archer, and R. Evans, Phys. Rev. E 71, 027401 (2005); J. Chem. Phys. 124, 054503 (2006).

${ }^{46}$ A. J. Archer and R. Evans, Phys. Rev. E 64, 041501 (2001).

${ }^{47}$ C. Grodon, M. Dijkstra, R. Evans, and R. Roth, J. Chem. Phys. 121,
7869 (2004); Mol. Phys. 103, 3009 (2005).

${ }^{48}$ M. Seul and D. Andelman, Science 267, 476 (1995).

${ }^{49}$ Z. Nussinov, J. Rudnick, S. A. Kivelson, and L. N. Chayes, Phys. Rev. Lett. 83, 472 (1999).

${ }^{50} \mathrm{~A}$. Ciach (private communication).

${ }^{51}$ A. Ciach and O. Patsahan, Phys. Rev. E 74, 021508 (2006).

${ }^{52}$ S. A. Brazovskii, Sov. Phys. JETP 41, 85 (1975).

${ }^{53}$ O. Patsahan and A. Ciach (unpublished). 